\title{
Tratamiento del reflujo vesico-ureteral primario en la infancia: comparación de dos revisiones sistemáticas
}

\author{
E. de la Peña Zarzuelo
}

Fundación Hospital Alcorcón. Madrid.

Actas Urol Esp 2005; 29 (2): 138-162

\section{RESUMEN}

TRATAMIENTO DEL REFLUJO VESICO-URETERAL PRIMARIO EN LA INFANCIA: COMPARACIÓN DE DOS REVISIONES SISTEMÁTICAS

Muchas prácticas médicas se continúan realizando sin conocer su efectividad ni el impacto real que tienen en la salud de los pacientes, por lo tanto es necesario fundamentar las recomendaciones profesionales en la evidencia científica.

Propósito principal: realizar una revisión sistemática (RS) sobre el manejo terapéutico del reflujo vésico-ureteral primario (RVUP) en urología pediátrica.

Objetivos: 1. Definir un tratamiento ideal para cada grado de RVUP, 2. Aclarar incertidumbres sobre diversos aspectos no abordables mediante estudios aislados.

Material y métodos: Se ha realizado una RS de los artículos aparecidos en todas las bases de datos disponibles, aplicando unos criterios de inclusión y exclusión de calidad mínima imprescindible.

Resultados y discusión: Tras la lectura crítica de más de 320 artículos y el estudio estadístico correspondiente de los datos agrupados, según el tipo de tratamiento y beneficios aportados, así como sus efectos indeseables y comparado nuestras conclusiones con las aportadas por el trabajo recogido recientemente en The Chocrane Library, hemos alcanzado las siguientes conclusiones: El tratamiento médico y el quirúrgico presentan la misma efectividad en la resolución de los grados I, II y III de RVUP, recomendándose el primero de ellos como tratamiento inicial tras el diagnóstico. El manejo endoscópico es igual de efectivo que la cirugía abierta, con menores efectos secundarios, sin haber encontrado diferencias entre las distintas sustancias analizadas para la inyección. Para los reflujos de alto grado IV y V no existen evidencias suficientes que aconsejen o desaconsejen cualquiera de los tratamientos. El tratamiento quirúrgico abierto, para cualquier grado de RVU, sólo presenta superioridad con respecto al tratamiento médico en el número de episodios de pielonefritis aguda durante el seguimiento. Esta afirmación no es posible aplicarla para el tratamiento endoscópico.

Palabras clave: Reflujo vesicoureteral. Revisión sistemática. Tratamiento. Infancia.

\section{ABSTRACT}

\section{PRIMARY VESICOURETERAL REFLUX TREATMENT IN CHILDHOOD: COMPARASION OF TWO SYSTEMATIC} REVIEW

Many medical practices are being carried out unawares of their efficiency, or of their actual impact on the health of the patients, therefore it is necessary to consider the support of professional recommendations with scientific evidence.

The primary objective: to perform a systematic review (SR) of the therapeutic management of primary VUR in pediatric urology.

Material and Methods: A systematic review has been performed, including scientific evidence-based medicine criteria, of the articles published in all of the available databases. Inclusion criteria concerning basic quality of the articles were considered essential, as well as exclusion criteria to be able to reject the articles.

Results and discussion: Subsequently, and following the critic reading of greater than 320 articles, statistical study of the grouped data was performed according to the type of treatment and to the benefits contributed by each treatment, and also to their undesirable effects. Finally we have made a comparison between our results and recent Cochrane Systematic Review. The following Conclusions were drawn from the results obtained and from the analysis of the texts. Both medical and surgical treatment present with similar effectiveness concerning resolution of grades I, II and III of VUR, and the former one is the recommended initial treatment following diagnosis. Endoscopic treatment is exactly as effective as open surgery for grades I, II and III with fewer undesirable effects secondary. There are no differences concerning the efficacy of the different injected substances. Not enough evidences exist for degrees IV and V that may recommend or advise against any of the treatments. In any degree of VUR, open surgical treatment is superior as far as medical treatment is concerned only regarding the number of acute pyelonephritis episodes during followup. This conclusion cannot be applied on endoscopic treatment. 
$\mathrm{E}^{1}$ avance del conocimiento científico no sólo debe realizarse a partir del descubrimiento de nuevas tendencias, la formulación de teorías inéditas o la génesis de conceptos originales. Lo que hoy damos por seguro, mañana es rebatido, y a fuerza de buscar lo último, olvidamos fundamentar lo primario. Debemos contemplar la acumulación de experiencia y el análisis razonado de lo ya estudiado. La lectura crítica y la aplicación de la metodología de investigación en los estudios realizados sobre una materia, aclara el estado de la cuestión y puede filtrar la realidad de los sesgos que la confunden. El número de publicaciones y artículos de revistas se multiplica exponencialmente y las fuentes de información médica crecen a diario. Lo publicado no reúne en ocasiones el nivel de calidad necesario y en otras los resultados ofrecidos pueden llevar a conclusiones inconsistentes, cuando no contradictorias. Precisamos de una herramienta de trabajo que nos ayude a refinar nuestras fuentes de información: un instrumento objetivo, riguroso, con método científico, reproducible y que aporte información de interés y utilidad clínica para la toma de decisiones en la práctica diaria. Las RS, alejadas de las clásicas revisiones bibliográficas, aplican el método científico caracterizado por la sistematización y por ende la reproducibilidad, aplicando técnicas estadísticas que combinan y resumen resultados de estudios previos. Las RS surgen como respuesta a la necesidad de contestar a los múltiples interrogantes con los que se enfrenta el médico en su práctica clínica diaria y se han propuesto como método para sintetizar y evaluar la evidencia clínica ${ }^{1} \mathrm{El}$ meta-análisis, como forma de aportar los resultados cuantitativos, permite integrar de forma eficiente toda la información válida existente y proporcionar suficientes datos para la toma racional de decisiones $^{2}$.

El RVUP constituye un problema importante dentro de la práctica urológica pediátrica. La profusión de estudios y la diversidad de opiniones sobre su manejo lo caracterizan. Lejos de implementar el conocimiento sobre el tema con una nueva serie más o menos larga de pacientes tratados, la justificación de nuestro trabajo se fundamenta en la búsqueda de la evidencia sobre este tema concreto. En fechas próximas a la lec- tura como tesis doctoral de este trabajo se recoge en The Cochrane Library un trabajo similar en su metodología, discusión y conclusiones, con el que deseamos compararnos, y que no pudo ser evaluado en nuestra RS.

\section{Estrategia de la búsqueda}

Se hicieron búsquedas en MEDLINE (1966septiembre 2003), EMBASE (1988-septiembre 2003), listas de referencias de artículos, búsqueda manual de literatura gris y guías clínicas de asociaciones, revisiones en el registro Cochrane de RS y de Ensayos Controlados y búsqueda de ensayos adicionales en las listas de referencias de los artículos relevantes, utilizando el Sciencie Citation Index.

De todos los artículos encontrados tras realizar la búsqueda sistemática, se hizo una primera selección descartando aquellos que no se ajustaron por su título, o por el desarrollo de su abstract, utilizando los criterios de inclusión que aparecen en la Tabla 1. Sobre estos se estableció un segundo filtro según los criterios de exclusión que aparecen en la Tabla 2. Los artículos que cumplieron satisfactoriamente los criterios señalados en los apartados anteriores fueron seleccionados para recoger su información de forma análoga a la recogida de datos en los pacientes individuales de un estudio clínico o epidemiológico, según los principios de objetividad, fiabilidad, ausencia de sesgos y precisión ${ }^{3}$.

\section{Limitaciones de nuestra búsqueda}

Se han descartado numerosos estudios debido a varias causas: recogida inconsistente de los datos, tiempo limitado de seguimiento, descripción

\section{Tabla 1}

Criterios de inclusión de revision de articulos

1. Artículos (no resúmenes) cuyo idioma original fue inglés o español o francés.

2. En los que se hubiera incluido un número mínimo de 20 pacientes.

3. Estudios referidos a RVU primario, no tratados previamente,

4. Debía aparecer claramente especificado cuál era el tratamiento realizado y el periodo de seguimiento

5. Se debía demostrar la ausencia de RVU postratamiento mediante cistografia, isotópica y/o convencional. 
Tabla 2

Criterios de exclusion de revision de articulos

1. Estudios duplicados o desfasados por otros posteriores.

2. Los que no contenían información suficiente para comparar entre las diferentes opciones terapéuticas.

3. Si no presentaban información detallada sobre la metodología empleada para la obtención de imágenes posttratamiento, el modo de analizarlas, o sobre el tipo de co-morbilidad asociada (infección urinaria, inestabilidad vesical, malformaciones).

4. Los abstracts de presentación de comunicaciones o pósters a congresos, por la falta de datos en los mismos y/o por una metodología inadecuada.

5. Cartas, editoriales, casos clínicos, datos no publicados

6. Estudios en los que no se especificaba claramente la técnica quirúrgica realizada, el tratamiento antibiótico administrado, el tiempo del mismo, o la sustancia endoscópica inyectada.

7. Estudios de validación de una técnica, ensayos de laboratorio, estudios sin resultado post-tratamiento.

8. Reflujos vésico-ureterales secundarios.

9. Estudios de revisión de expertos o financiados por la industria farmacéutica.

incompleta de los tratamientos utilizados, mala definición de la población objeto de estudio, etc., pero de manera significativa nos gustaría destacar los siguientes hechos:

Existen pocos estudios controlados, randomizados y prospectivos que comparen el tratamiento médico con el quirúrgico. Se han descrito varias escalas que clasifican el grado de RVU, al menos 5. La más empleada es la International Study Classification; sin embargo, numerosos trabajos se refieren a clasificaciones anteriores o incluso agrupan el RVU en grado bajo, intermedio y alto, lo cual dificulta enormemente la comparación entre estudios. Así mismo muchos estudios además de no aportar claros resultados post-tratamiento, agrupan estos en función de la clasificación arriba mencionada: reflujos de bajo, intermedio y alto grado. Aunque el reflujo es diagnosticado con más frecuencia en las niñas que en los niños, las secuelas pueden ser diferentes entre estos. La mayoría de los resultados no aportan datos referidos al sexo de los pacientes. La posibilidad de afectación ureteral bilateral, con diferente grado de reflujo en cada unidad complica la comparación entre grupos, incluso del mismo tipo de tratamiento utilizado. En gene- ral los tiempos medios de seguimiento en los pacientes intervenidos, bien mediante reimplante, bien mediante cirugía endoscópica, es en general menor que los pacientes sometidos a tratamiento antibiótico, por lo tanto se explica que las complicaciones aparecidas y los resultados inferidos sean diferentes. En la mayoría de las series de tratamiento antibiótico analizadas no se realiza un análisis separado por tiempo de tratamiento y se agrupan todos los pacientes en un conjunto de eficacia/tiempo de seguimiento de la revisión de datos. Por fin, durante los últimos años ha proliferado una variedad importante de sustancias utilizadas para la inyección submeática, lo cual representa un sesgo en el análisis de los resultados del tratamiento endoscópico, además reduce de manera considerable los estudios comparativos de series de tamaño estadísticamente significativo.

\section{Conflicto de intereses}

Nuestro trabajo no presenta en su génesis ni desarrollo ningún potencial conflicto de interés.

\section{ANTECEDENTES}

La importancia del RVU, una de las patologías más frecuentemente tratadas por el urólogo pediátrico, radica en los siguientes hechos: aproximadamente un tercio de los niños con infecciones urinarias febriles padecen $\mathrm{RVU}^{4}$, esto tiene especial importancia si se tiene en cuenta que entre el 3\% y el 5\% de las niñas y el $1 \%$ y $2 \%$ de los niños padecen infecciones urinarias antes de la pubertad ${ }^{5-7}$. La incidencia de infección urinaria antes de los seis años ha sido definida entre las niñas en un 6,6\% y entre los niños en un $1,8 \%^{8}$. De las asistencias de los pediatras a los niños menores de tres meses, las infecciones urinarias, constituyen las infecciones bacterianas más frecuentes ${ }^{9}$ y el $6 \%$ aproximadamente de los procesos febriles en la infancia ${ }^{10-12}$ La incidencia de reflujo se recoge en la Tabla 3.

Se denomina RVUP aquel que está producido por la disfunción de la unión uretero-vesical secundaria a una alteración congénita que implique una longitud insuficiente del trayecto transmural y submucoso del uréter y una lateralización intravesical del mismo. Cuando el RVU se 
Tabla 3

Incidencia de RVU diferentes grupos de pacientes

\begin{tabular}{lcc}
\hline Grupo & \% de RVU & Referencias \\
Bacteriuria asintomática & 29,8 & {$[215-217]$} \\
Infección urinaria simple & 31,1 & {$[4,11,218-226]$} \\
Infección urinaria febril & 29,0 & {$[227-230]$} \\
Pielonefritis & 31,5 & {$[73,231-233]$} \\
Infección urinaria <1 año & 31,0 & {$[4,11,220,231]$} \\
Infección urinaria 1- 5 años & 31,6 & {$[11,218,228,231]$} \\
Infección urinaria >5 años & 30,3 & {$[11,231]$} \\
Infección urinaria en niños & 30,0 & {$[11,226,231]$} \\
Infección urinaria en niñas & 32 & {$[11,226,231]$} \\
\hline
\end{tabular}

asocia a alteraciones anatómicas o funcionales que aumentan la presión intravesical o el mecanismo valvular ureteral ha sufrido alteraciones iatrogénicas o forma parte del contexto de una malformación congénita hablamos de RVU secundario.

En 1981 el International Reflux Study Comité $^{13}$ establece la clasificación internacional hoy en día utilizada y popularizada, que clasifica el reflujo en función de la cantidad de contraste que se visualiza en el tracto urinario superior tras la realización de una cistografía convencional (cistograma radiológico), tal y como queda resumido en la Tabla 4 y esquematizado en la Figura 1. En la actualidad la ecografia prenatal puede diagnosticar precozmente el RVU por la dilatación del tramo urinario superior, correspondiendo aproximadamente entre el 25-30\% de las hidronefrosis prenatales a RVU ${ }^{14-19}$. El diagnóstico de RVU está basado en criterios de imagen. Bien la cistografía convencional, bien la isotópica o los menos frecuentemente utilizados sistemas de eco-cistografía ${ }^{20-21}$ (Fig. 2)

Las consecuencias más importantes derivadas del RVU vienen expresadas en la cadena de acontecimientos que se inicia en la infección urinaria, la pielonefritis, la cicatriz y el daño renal y finalmente la hipertensión, la alteración de la función renal y el posible desarrollo subsiguiente de insuficiencia renal crónica terminal que precise de diálisis o trasplante renal. Además el RVU es causa de morbilidad gestacional y perinatal ${ }^{22} \mathrm{y}$ de alteración del crecimiento renal y pondoestatural $^{23}$.
Tabla 4

Clasificación y esquema del RVU según el Internacional Reflux Study Committee ${ }^{13}$

\section{Grado de reflujo Descripción}

I Aparición de contraste en un uréter no dilatado.

II El contraste aparece en el uréter, pelvis renal y cálices, sin dilatación

III Dilatación leve o moderada del uréter, la pelvis y los cálices con preservación de los fórnix

IV Moderada dilatación uretero-pielo-caliciliar, con cierto grado de tortuosidad

V Gran dilatación uretero-pielo-caliciliar, con tortuosidad y pérdida de las impresiones papilares por dilatación calicicliar

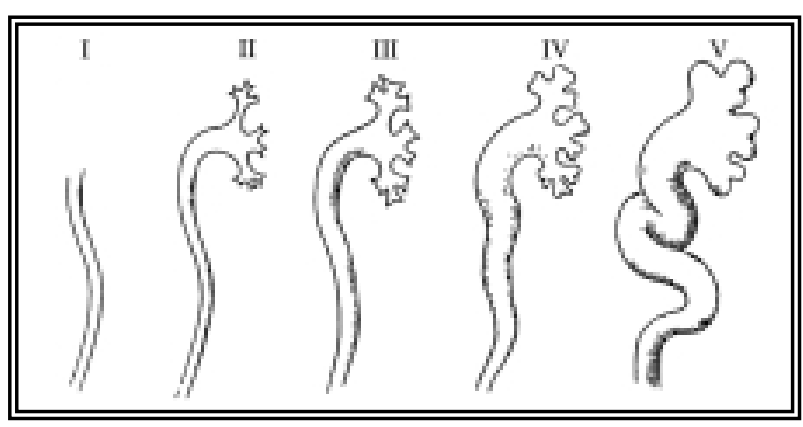

FIGURA 1

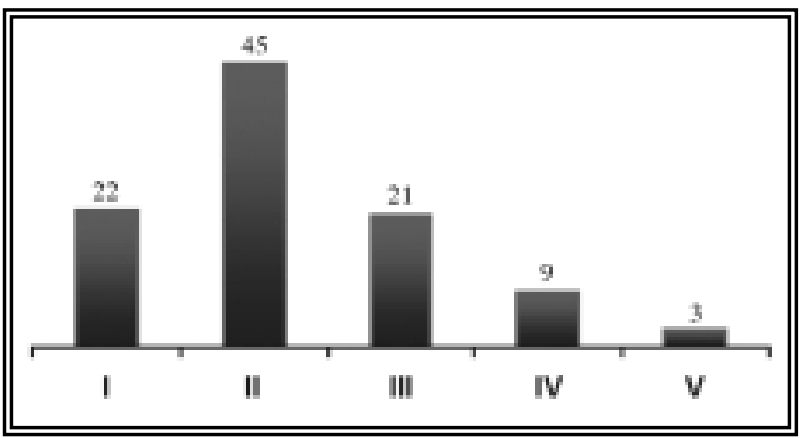

FIGURA 2. Distribución porcentual del RVU según el grado al diagnóstico $[25,32,55,56,59,81,183,234-243]$

El objetivo primordial del tratamiento por lo tanto es prevenir las pielonefritis y el daño renal:

A lo largo del tiempo y gracias a la aparición de distintos fármacos (anticolinérgicos), la descripción de diferentes técnicas quirúrgicas, el incremento de sustancias utilizadas para el tratamiento endoscópico en busca de una terapia quirúrgica mínimamente invasiva, o la descripción de los hallazgos urodinámicos que ayudaban a entender la etiopatogenia del reflujo, hemos podido constatar la existencia de varias líneas $\mathrm{u}$ 
opciones de tratamiento del RVUP (Tabla 5). Todas ellas pueden ser clasificadas en dos grandes grupos:

1. El tratamiento médico está basado en el principio de que el RVU presenta con frecuencia una resolución espontánea y que la morbilidad o las complicaciones del mismo pueden prevenirse sin un tratamiento invasivo.

2. La base del tratamiento quirúrgico es reconocer las situaciones o pacientes que presentan un riesgo potencial significativo de padecer daño renal $\mathrm{u}$ otras complicaciones asociadas al reflujo. En estos casos son asumibles los efectos indeseables de la cirugía.

Tabla 5

Opciones terapéuticas para el RVUP

1. Vigilancia y tratamiento antibiótico intermitente (frente a infecciones urinarias)

2. Entrenamiento miccional (que incluye micción pautada y reeducación miccional)

3. Profilaxis antibiótica nocturna -quimioprofilaxis(QMP)(continua y a $1 / 3$ de la dosis terapéutica)

4. Combinación de QMP y entrenamiento miccional

5. $\mathrm{QMP}+$ anticolinérgicos (inestabilidad vesical) + entrenamiento miccional

6. Tratamiento quirúrgico (que incluye cualquier modificación de la unión uretero-vesical)

7. Tratamiento endoscópico

\section{OBJ ETIVOS}

El propósito principal del presente trabajo es realizar una RS sobre el manejo terapéutico del RVUP en la población pediátrica, cubriendo los siguientes tipos de tratamientos: Tratamiento farmacológico, mediante quimioprofilaxis antibiótica; tratamiento quirúrgico mediante reimplante ureteral y tratamiento endoscópico mediante la inyección submeática.

Además se desean alcanzar los siguientes objetivos secundarios: Obtener un estimador promedio ideal (tratamiento ideal) a partir de las estimaciones cuantitativas y cualitativas de los estudios individuales y comparativos para cada grado de reflujo vésico-ureteral; y aclarar incertidumbres sobre diversos aspectos de la patología no abordables desde la perspectiva de estudios aislados, pero que pueden examinarse en un contexto de meta-análisis.

\section{RESULTADOS Y DISCUSIÓN: ANÁLISIS DE DATOS EN RELACIÓN A LA OPCIÓN TERAPÉUTICA}

1. DISMINUCIÓN DEL GRADO DE REFLUJO Tratamiento médico

Hemos analizado 26 artículos de los que se pueden extraer resultados significativos. Los problemas encontrados en la revisión de estos trabajos han sido la dificultad para establecer similitudes dentro del concepto de "éxito" (resolución completa del RVU vs. disminución del grado de éste). Algunos de estos trabajos se refieren al éxito por unidad renal y otros al éxito por paciente. Por último resultó dificultoso extraer conclusiones de la tasa de resolución por año de seguimiento, pues en la mayoría de los casos el resultado es expresado de forma global según el tiempo medio de seguimiento para toda la serie. Otras series por su antigüedad no hacen referencia exacta a la clasificación internacional del RVU.

Se establecieron puntos de corte arbitrarios en la edad de los pacientes para poder clasificarlos en intervalos: menores de dos años, los diagnosticados de esta edad hasta la preescolar (5 años) y aquellos diagnosticados durante la edad escolar (5 a 10 años). No hemos encontrado trabajos significativos que analicen adecuadamente las diferencias entre uni y bilateralidad para los RVU de bajo grado (I y II).

Se ha definido la edad media de desaparición del RVU entre los 4,6 y los 6,8 años24-26.Estas cifras no son igualmente aplicables en los dos sexos. Así está descrito como para grados bajos (I y II) en niñas de 10 años de edad se resolverán espontáneamente casi la totalidad de los casos antes de los 13 años, sin embargo solamente el $50 \%$ de los niños cumplirán esta misma condición ${ }^{27}$ Para los grados III y IV no se observará resolución espontánea de casi ningún caso a esta edad $^{28}$. Basado en los estudios de Arant, Skoog, Belman y Tamminen ${ }^{24,}$ 29-31, Elder ${ }^{32}$ ha publicado las curvas de resolución para los grados I y II y IV, y para el grado III ajustado a la edad del diagnóstico.

\section{Tratamiento quirúrgico abierto}

Se incluyeron aquellos procedimientos técnicos que por su representatividad o por el número de pacientes incluidos en la serie constituían una 
muestra representativa, aplicando igualmente los criterios de inclusión conocidos para esta revisión así como en los casos en los que era factible, las complicaciones derivadas de dicho procedimiento. Los datos han sido agrupados por técnicas quirúrgicas y a su vez disociados según el grado de reflujo tratado. No fueron tenidas en cuenta las variaciones personales a las técnicas quirúrgicas estándar. Los estudios seleccionados correspondieron a 13 que utilizaron el reimplante transtrigonal de Cohen, 5 que usaron el de Gil-Vernet, 13 el de Lich-Gregoir, y 16 usaron el procedimiento de Politano-Leadbetter. El resultado final del procedimiento quirúrgico se pudo obtener en $94,9 \%$ de los pacientes analizados, especificado en 4.556 tratamientos exitosos de los 4.691 uréteres tratados $(97,1 \%)$. No en todos los trabajos analizados se obtienen resultados individualizados en función del grado de RVU que fue tratado, sin embargo se obtuvieron suficientes casos que permiten sacar conclusiones con alto grado de evidencia. Para el grado I de las 88 unidades renales tratadas el resultado fue efectivo en todas ellas, para el grado II el resultado fue exitoso en 723 de las 730 unidades tratadas (99\%), en el grado III 757 de las 765 uréteres operados corrigieron posteriormente el reflujo (98,9\%), en el grado IV 242 de los 249(97,1\%) y en el grado V 72 de los 79 operados (91\%). Merece la pena considerar sin embargo que el resultado en grados severos varía mucho de unas series a otras del $25 \% 33$ al $100 \%^{34}$ y que se ve muy influido por el tipo de técnica, habiéndose encontrado un importante número de artículos en los que se asociaba a las técnicas de reimplante algún tipo de "modelaje ureteral", no precisándose en muchos de estos trabajos si se correspondían a casos de RVU de alto grado o megauréteres refluyentes, incluyéndose en estos casos las técnicas clásicas de Starr ${ }^{35}$, Kalicinsk ${ }^{36}$ o Hendren ${ }^{37}$. En general podemos afirmar que el resultado medio del tratamiento quirúrgico es del $97 \%$, siendo de mayor efectividad para los grados I-III, y algo menor para el grado IV, aunque con valores por encima del 95\%. Los grados V tienen un peor resultado, aunque por encima del 90\%. Cuando se asocia técnica de modelaje por asociación de megauréter y reflujo este baja hasta el $87,2 \%$.

\section{Tratamiento endoscópico}

Se han analizado veintitrés trabajos referidos al tratamiento endoscópico de reflujo tras aplicar los criterios de inclusión y exclusión ${ }^{38-61}$ en los que se analizan las cinco substancias utilizadas con mayor número de individuos y periodos de seguimiento más largo: polidimetilsiloxano (Macroplastico ${ }^{\circledR}$ ),cinco trabajos, uno de ellos comparativo randomizado de macroplastico frente a cirugía extravesical; politetrafluoruro de etileno (Teflon ${ }^{\circledR}$ y Politef ${ }^{\circledR}$ ), 12 trabajos, los de más largo seguimiento, uno de ellos multi-institucional e internacional; dextranomero del copolímero del ácido hialurónico (Deflux ${ }^{\circledR}$ ), tres trabajos, uno de ellos comparado con macroplastico en estudio prospectivo y randomizado; Colágeno bovino purificado (Zyplast ${ }^{\circledR}$ ), tres trabajos en RVU primario y condrocitos autólogos en un trabajo de seguimiento a largo plazo. Todos ellos se utilizaron en series de individuos con similar distribución de pacientes, edades y sexo. Los seguimientos medios variaron entre 1 año y 17 , siendo la media superior a 4 años. En todos se aplicó la técnica endoscópica descrita por O`Donnell y Puri62 Analizaremos las tres sustancias más frecuentemente utilizadas.

Politetrafluoruro de etileno (Teflon ${ }^{\circledR}$ )

La primera descripción en la literatura sobre el uso de esta sustancia fue realizada en $1984^{62}$, desde entonces su manejo se ha popularizado y las series han alcanzado un tiempo medio de seguimiento en algún caso superior a 14 años. Las indicaciones para su uso han crecido progresivamente, lo que ha provocado un aumento exponencial del número de publicaciones, no siempre con los criterios de calidad que se han tenido en cuenta para su inclusión en el presente análisis. La mayor parte de ellos aportan un mayor número de pacientes a la serie global de tratados, aludiendo únicamente al grado de resolución de reflujo y con un escaso tiempo de seguimiento, tal es el caso de los trabajos de Schulman $^{57}$ Dodat $^{55}$, Davies ${ }^{58}$ Lacombe $^{54}$ Vereecken $^{53}$ o Sauvage ${ }^{56}$. El porcentaje de infección urinaria postratamiento se estableció en un 2,3-3,6\%. Del total de las series el 2,4\% [IC: 95\%, 0,9-4,5\%] requirió solucionar el reflujo mediante reimplante ureteral abierto. En ninguno de los trabajos 
realizados se hace mención al fracaso de la técnica por la asociación a vejiga hiperactiva (VHA), mal llamada disfunción miccional, entendida esta únicamente como alteraciones clínicas (urgencia, urgencia incontinencia, aumento de la frecuencia miccional o dificultad de vaciado). Estos son los únicos criterios encontrados en la totalidad de los textos revisados para el tratamiento endoscópico, asumibles como VHA, pero sin comprobación urodinámica. A nuestro juicio, se hace imprescindible un trabajo que aplique criterios urodinámicos para interpretar los fallos asociados a esta técnica.

\section{Polimetilsiloxano (Macroplastico ${ }^{\circledR}$ )}

Se han analizado 4 trabajos que cumplían con los criterios de inclusión y exclusión adoptados en nuestra revisión. Los tiempos de seguimiento son, en comparación con el Politetrafluoruro de etileno más cortos, ya que ha sido incorporado al tratamiento del RVU más tarde. En este caso se ha descrito una asociación entre el fallo de la inyección y la coexistencia de alteraciones funcionales vesicales entre el 45 y el $50 \%{ }^{44,45}$. Herz ${ }^{45}$ describe la aparición de un $5 \%$ de reflujo contralateral tras la primera inyección; así como un $4 \%$ de reintervenciones por vía abierta tras fracaso de la inyección.

Dextranomero del copolímero del ácido hialurónico (Deflux $\left.{ }^{\circledR}\right)$

Pocos trabajos recogen con largo tiempo de seguimiento los efectos del Deflux ${ }^{\circledR}$ en el tratamiento endoscópico del reflujo, en total tres trabajos cumplieron los criterios de inclusión aportando unos resultados ligeramente inferiores a los dos productos analizados previamente, sin poder llegar a la significación estadística. Este hecho es indicado por Oswald ${ }^{52}$ (Macroplastico® frente a Deflux $\left.{ }^{\circledR}\right)$, tanto de forma inmediata ( $86,2 \%$ vs. $71,4 \%$ de éxito respectivamente), como al año de seguimiento $(80,9 \%$ vs $67,6 \%$ de persistencia del éxito respectivamente). Capozza ${ }^{63}$ interpreta la mayoría de los fallos en el contexto de una disfunción vesical presente en el $26 \%$ de estos casos, con un resultado final de éxito sobre 320 unidades del $84 \%$. Lackren ${ }^{64}$ con un seguimiento de cinco años sobre 221 pacientes (338 unidades renales), presenta unos resultados exi- tosos del $77,5 \%, 77,8 \%, 62,5 \%$ y $66,6 \%$ para los grados II, III, IV y V respectivamente; resultados que obtiene en el $81 \%$ de la primera inyección que se incrementan con el $43 \%$ de la segunda y el $50 \%$ de la tercera.

\section{CICATRICES RENALES}

La correcta identificación de las pielonefritis agudas en los niños, la afectación y extensión del daño renal causado y la determinación de la posibilidad de reinfección siguen siendo fundamentales para determinar los riesgos a largo plazo, y las posibilidades de desarrollar hipertensión arterial e insuficiencia renal en el futuro. Los aspectos diagnósticos de las pielonefritis agudas y de la afectación renal han generado numerosos trabajos, con grandes discrepancias entre ellos. Los primeros artículos aparecidos en los que se determina la existencia de cicatriz renal post-pielonefritis, anteriores a la década de los ochenta, están basados en estudios urográficos, diagnosticándose daños renales permanentes en un 5-20\%65. Actualmente y gracias a los estudios con DMSA (ácido dimercaptosuccinio) marcado con Tc99m se diagnostican un $41 \%$ de lesiones renales permanentes en riñones que han padecido pielonefritis $^{66}$. La ecografía renal presenta una baja sensibilidad 40\%, según Nguyen ${ }^{67}$. En la gammagrafía renal con DMSA, el marcador se incorpora en un $60-70 \%$ a los túbulos renales y sólo un $2-5 \%$ es excretado por la orina, de tal manera que da una valoración fiel de la función global y relativa de cada unidad renal, habiéndose determinado su sensibilidad y especificidad para el diagnóstico de cicatriz renal tras pielonefritis en el 87 y $100 \%$ respectivamente ${ }^{68-69}$. Las alteraciones en el DMSA (hipocaptación del trazador) después de un episodio febril en niños con un rango de edad de 9 a 21,7 meses oscila entre el 52 y el 92\%, aunque posteriormente sólo quedan cicatrices permanentes en un $15-41 \%^{70-73}$. La gammagrafía renal además diagnostica la cicatriz renal tanto en niños con reflujo demostrado, como en aquellos en los que no se identifica este mediante cistografia miccional, aunque existe asociación significativa entre el grado de reflujo y la aparición de cicatrices ${ }^{73-77}$. De acuerdo con esto podemos considerar que el RVU es un factor crítico para la aparición de cicatrices renales, pero no el único. 
Estos hechos han sido considerados por algunos autores lo suficientemente significativos como para plantear la gammagrafía renal con DMSA Tc99m como prueba inicial e incluso única en el diagnóstico de un episodio de infección urinaria febril en el niño ${ }^{78,79}$.

La prevención de cicatrices renales es pues el principal objetivo en el tratamiento del RVU, debiéndose analizar con profundidad. Se han estudiado 5 trabajos prospectivos y randomizados que comparan los resultados del manejo terapéutico, médico o quirúrgico, con respecto a la aparición de nuevas cicatrices renales. En 4 de ellos estas se determinaron mediante urografía intravenosa y en uno de ellos con gammagrafía renal ${ }^{80-84}$. Sin embargo, la mayoría de los trabajos hacen referencia a descripciones individuales de una de las dos ramas de tratamiento, por lo que hemos realizado las comparaciones de las dos con aquellos grupos de similar distribución de casos, grados y tiempo de seguimiento, obteniendo riesgos relativos combinados de las diferentes series. Se han analizado 15 trabajos de tratamiento médico y 15 de tratamiento quirúrgico, que incluyen 5 de tratamiento endoscópico, cuyas dos substancias empleadas fueron el Teflon ${ }^{\circledR}$ y el Macroplastico ${ }^{\circledR}$. Los trabajos que hacen referencia a estudios prospectivos y randomizados entre tratamiento quirúrgico y médico no muestran diferencias estadísticamente significativas. Todos los trabajos comparan cirugía abierta frente a tratamiento médico con quimioprofilaxis. No se han encontrado estudios randomizados prospectivos que comparen el tratamiento endoscópico con el médico. Sobre un total de 806 niños (453 operados y 353 de tratamiento médico), seguidos entre 3,4 y 5 años, todos con grados de RVU de III a V, el tratamiento médico fue ligeramente superior al quirúrgico pero sin alcanzar la significación estadística ( $11,3 \%$ frente a $12,6 \%$ de nuevas cicatrices). Merece la pena destacar, sin embargo que la aparición de estas, se retrasa más en el tiempo en los pacientes tratados con quimioprofilaxis. El $80 \%$ de las lesiones en el grupo de tratamiento quirúrgico se producen en los 10 meses siguientes a la cirugía, mientras que en el grupo de tratamiento médico aparecen a lo largo de los 5 años posteriores al seguimiento ${ }^{81}$. Son conocidas las profundas dis- crepancias encontradas entre las dos ramas, europea $^{82}$ y americana ${ }^{83}$, del Estudio Internacional sobre Reflujo. Se analizó la frecuencia de aparición de nuevas cicatrices tras tratamiento, siendo el 15,7\% (médico) y 17,2\% (quirúrgico) en la rama europea y el $21,5 \%$ (médico) y $31,4 \%$ (quirúrgico) en la Americana. Estos datos han sido posteriormente corroborados por Piepsz ${ }^{85}$, sobre un grupo randomizado y prospectivo, seguido durante cinco años, de pacientes afectos de RVU de alto grado, $82 \%$ de los cuales presentaban alteraciones previas al inicio del tratamiento en el DMSA, sin encontrar diferencias significativas entre la opción médica y quirúrgica. Los 15 estudios no randomizados, referidos a los resultados de la opción médica (quimioprofilaxis), combinados entre si, muestran un riesgo relativo del 4,1\% [0 -24,7\%, I.C.:95\%] ${ }^{29,81,86-98}$ Los 11 trabajos que analizan la aparición de nuevas cicatrices renales tras tratamiento quirúrgico, se refieren a procedimientos de reimplante ureteral transvesical ${ }^{81,8489,99.106}$, y presentan unos porcentajes medios del 4,6\% [0-16,7\%, I.C.:95\%]. Se han analizado 6 trabajos que de forma individual describen la aparición de nuevas cicatrices tras tratamiento endoscópico $40,41,47,4952,107$, siendo la media de 5,2\% [0-12,5\%, I.C.:95\%].

\section{CRECIMIENTO RENAL}

La impresión clínica, basada en algunos trabajos publicados en la década de los $70^{108-109}$, de que el RVU está asociado al retraso en el crecimiento renal, especialmente en los casos de RVU de alto grado ${ }^{110,111}$ ha motivado el análisis ulterior de esta variable, comparando las diferentes opciones terapéuticas. Sin embargo, la determinación del tamaño renal está afectada por algunos problemas, incluso en los individuos normales. Las pruebas utilizadas para su medición están sujetas a numerosas subjetividades y limitaciones técnicas. La ecografía, si bien se considera una buena técnica para la medición de órganos intrabdominales, está influida por la experiencia del examinador. No queda claro tampoco que el tamaño renal esté íntimamente relacionado con su función. No existe un patrón de la normalidad morfológica renal, existen riñones largos y delgados y otros cortos y gruesos, con la misma masa nefronal en definitiva. 
El tamaño renal no depende únicamente del crecimiento del riñón afecto sino también del mecanismo compensador que establece el riñón contralateral sano, de las malformaciones congénitas asociadas, pero no producidas por el RVU, del número de episodios de infecciones urinarias sucedidas previas al diagnóstico de RVU, y a los cambios por nefropatía asociada al reflujo estéril prenatal $^{112}$, especialmente en niños con reflujo de alto grado. La mayoría de los artículos encontrados que hacen referencia a la influencia que sobre el tamaño renal tiene cada tipo de tratamiento analizan series retrospectivas sin un apropiado grupo control ni seguimiento prolongado. Existen tres estudios que de forma prospectiva y randomizada, utilizan la urografía como método para determinar el tamaño renal, y con seguimiento superior a cinco años en dos de ellos $^{81-83}$, y superior a diez en el tercero ${ }^{113}$. Ninguno de ellos encuentra diferencias estadísticamente significativas a favor de ninguna de las dos opciones (médica o quirúrgica). No hemos encontrado ningún trabajo que de forma individual o comparada con tratamiento quirúrgico y/o médico analice los resultados del tratamiento endoscópico sobre el desarrollo renal. Las series de Chertin ${ }^{40,41}$ que ofrecen resultados a largo plazo (más de 10 años), en reflujos de alto grado, tratados mediante la inyección de politetrafluoroetileno (Teflon $\left.{ }^{\circledR}\right)$, sólo contemplan la resolución del reflujo.

\section{FUNCIÓN RENAL}

La influencia del tratamiento seleccionado en la mejoría o estabilización de la función renal presenta el inconveniente de no ser valorable si la afectación es unilateral, aunque esta fuese severa. Sólo la función renal alterada por afectación bilateral podría verse mejorada tras aplicar el tratamiento, y sólo cuando esta fuese severa ${ }^{114}$. Smellie ${ }^{115}$ realiza un estudio prospectivo y randomizado entre pacientes afectos de RVU de alto grado bilateral con nefropatía severa, para comparar el tratamiento médico con el quirúrgico. Clasifica a los pacientes por edad e índice de filtración glomerular. Establece un seguimiento medio de cuatro años, y en un grupo de 48 pacientes del total de la serie durante más de diez años. Tras estimar la función glomerular median- te aclaramiento, urografía y DMSA, no encuentra diferencias estadísticamente significativas entre los dos grupos. Analizados individualmente los dos tipos de tratamiento, de una parte Scott $^{103}$ describe mejoría en la función glomerular en los pacientes intervenidos mediante reimplante ureteral, especialmente en aquellos con afectación bilateral y cuya función glomerular pretratamiento era inferior a $50 \mathrm{ml} / \mathrm{min} / 1.73 \mathrm{~m}^{2}$. De otra parte Poulsen ${ }^{116}$ usando el mismo tipo de determinación para la función renal, obtiene los mismos resultados con tratamiento antibiótico independientemente del grado de reflujo. Igualmente en estudios controlados ${ }^{81,83}$ no se ha apreciado beneficio en la función glomerular tras aplicar tratamiento quirúrgico. Tampoco hemos encontrado trabajos que analicen este aspecto tras aplicar tratamiento endoscópico. Únicamente Läckgren $^{64}$ refiere no haber encontrado empeoramiento de la función renal dentro de los pacientes de su serie, sin aportar datos específicos a este respecto.

\section{INFECCIÓN URINARIA}

La experiencia clínica nos ha demostrado que los pacientes diagnosticados de RVU y que han sido sometidos a tratamiento, bien de quimioprofilaxis, bien quirúrgico, siguen presentando infecciones urinarias en el seguimiento de su patología. La importancia de estas infecciones es su relación directa con la aparición de nuevas cicatrices renales que puedan comprometer la función renal futura o derivar en una hipertensión arterial. Basados en estudios prospectivos que ya han sido analizados en el apartado correspondiente a cicatrices renales y opción terapéutica, hemos demostrado que no existen diferencias estadísticamente significativas a este respecto entre las dos opciones terapéuticas. Razonablemente si existe una relación directa entre las infecciones urinarias y la aparición de pielonefritis, y por lo tanto de daño real con cicatriz, no deberían existir tampoco diferencias significativas entre las dos opciones terapéuticas con respecto al número de infecciones urinarias. Con la utilización del DMSA como prueba sensible y específica de afectación renal se han aclarado aspectos que vienen a modificar algunas pautas de actuación terapéutica. No todas las infeccio- 
nes urinarias febriles comportan la aparición de una cicatriz renal, abriéndose así un nuevo abanico de patologías que afectando al tracto urinario superior y produciendo fiebre, no dañan al parénquima renal, tal es el caso de pielitis o ureteritis. Jakobson ${ }^{73}$ encuentra cambios en el DMSA en el 86\% de los pacientes en el momento agudo, disminuyendo al 59\% a los dos meses y al $37 \%$ a los dos años tras la infección. Además sólo el $25 \%$ de estos niños tenían asociado RVU, valores que se repiten para otros autores entorno al $39 \%{ }^{79}$. Estos hechos estarian relacionados con factores de adherencia uroepitelial del huésped o factores de virulencia del microorganismo. De otro lado, ni siquiera las imágenes de hipocaptación obtenidas en las fases agudas de la pielonefritis, visualizadas incluso a los tres meses de la misma, se confirman en análisis posteriores a los seis meses. En este caso hechos como el tratamiento precoz, incluyendo fármacos antiflamato$\operatorname{rios}^{117}$ disminuyen la progresión del daño renal. Se hace pues necesario determinar con evidencias científicas, la relación entre las infecciones urinarias, definida por bacteriuria con síntomas, y opción terapéutica para el RVU. En el brazo europeo del Estudio Internacional de Reflujo, aplicado a grados III y IV, no se encontraron diferencias estadísticamente significativas con respecto al número de infecciones urinarias, 39\% para la cirugía $38 \%$ para el tratamiento médico ${ }^{6}$. Se encontraron resultados muy similares en el brazo americano. Beetz ${ }^{118}$ en un estudio sobre 158 pacientes seguidos durante 20 años tras el reimplante ureteral (que en el $82 \%$ de los casos se habian descrito infecciones febriles preoperatorias y $18 \%$ infecciones urinarias afebriles), encuentra que tras la observación continuaron teniendo infecciones predominantemente afebriles un $66 \%$, incluidas el $74 \%$ de las mujeres. El riesgo relativo de padecer una infección urinaria post-tratamiento quirúrgico frente al médico es de 0,97 (0,79-1,19, I.C.:95\%) sin poder encontrar diferencias significativas entre ambas opciones terapéuticas ${ }^{32}$. Con respecto al riesgo de padecer una pielonefritis Jodal $^{6}$ encuentra un 9,3\% dentro de su serie quirúrgica, comparada con el $21 \%$ de la serie médica. Weiss ${ }^{83}$.encuentra datos muy similares $(7,8 \%$ vs. $22,1 \%)$. Elo $^{8}$ en un estudio de casos y controles sobre 40 pacientes encuentra un $72,5 \%$ dentro del grupo de controles (tratamiento médico) frente al $22,5 \%$ de los casos quirúrgicos. Hansson ${ }^{119}$ en un estudio de quimioprofilaxis con sulfadiacina-trimetropin encuentra un tasa de pielonefritis en los 860 meses de seguimiento del $27 \%$. Beetz ${ }^{118}$ en el estudio antes referido describe que los pacientes intervenidos por RVU siguieron manteniendo infecciones urinarias, afebriles en un $16 \%$ y febriles en un $38 \%$, el $82 \%$ de los cuales se repitieron más de una vez. Como nos recuerda Mansfield ${ }^{120}$ estas infecciones seguidas a tan largo plazo pueden verse sesgadas por la incidencia de infecciones urinarias en la mujer con el inicio de las relaciones sexuales. El riesgo relativo se situaría en un 0,39 $(0,26-0,58 \%$, I.C.: $95 \%)$ a favor del tratamiento quirúrgico. De los trabajos revisados sobre tratamiento endoscópico del RVU destacan los resultados ofrecidos por Lipsky ${ }^{47}$, con un $37 \%$ de infecciones urinarias tras inyección de colágeno a los 4 años de seguimiento; el 9\% de infecciones urinarias y el $3,5 \%$ de pielonefritis en una serie de 225 inyecciones de Deflux ${ }^{\circledR}$ de Lackren $^{64}$ y el $2,3 \%$ de infecciones urinarias de la amplia serie de Puri sobre 717 uréteres de alto grado ${ }^{41}$.

En definitiva en la comparación entre tratamiento médico y quirúrgico encontramos tras la revisión de la literatura tres hechos paradójicos:

1. No existe diferencias estadísticamente significativas con respecto al número de infecciones urinarias.

2. El tratamiento quirúrgico está relacionado con una menor incidencia de pielonefritis postoperatorias que el manejo médico con quimioprofilaxis.

3. Sin embargo, no existen diferencias estadísticamente significativas con respecto al desarrollo de nuevas cicatrices.

\section{HIPERTENSIÓN ARTERIAL}

Las cicatrices renales diagnosticadas en los niños afectos de RVU se producen como consecuencia de daño post-infeccioso al parénquima, displasia renal asociada al reflujo o ambos. Estas son la mayor causa de hipertensión arterial en el niño y en el adulto joven y son las responsables del 25\% de las insuficiencias renales terminales en niños en Gran Bretaña ${ }^{121}$ Se hace necesario determinar, a partir de los trabajos publicados, 
qué pacientes están en riesgo de padecer hipertensión arterial y/o insuficiencia renal. Cuáles son los factores de riesgo evitables de aparición de hipertensión e insuficiencia renal. Qué pruebas son necesarias solicitar durante el seguimiento y durante cuánto tiempo. Qué tipo de tratamiento se debe aplicar para cada caso con el fin de evitar la aparición de nuevas cicatrices y por lo tanto disminuir la probabilidad de padecer hipertensión arterial. Es imprescindible encontrar trabajos que analicen los diferentes factores de forma individual y multivariable y que posean un tiempo de seguimiento lo suficientemente amplio como para establecer conclusiones sólidas. Es fundamental establecer grupos de comparación homogéneos en cuanto al diagnóstico (grado de RVU), sexo, edad y tratamiento administrado. En este sentido no se ha encontrado ningún trabajo que haga referencia a las consecuencias que sobre la tensión arterial tiene el tratamiento endoscópico, por lo que todas las conclusiones se centran en la comparación del tratamiento médico con antibioterapia profiláctica frente al tratamiento quirúrgico abierto. Para el análisis de los resultados de hipertensión arterial se ha tenido en cuenta la definición y clasificación de hipertensión arterial dada por la Academia Americana de Pediatría, que se puede encontrar en la dirección: www.aap.org/policy/re9715t1.htm. Se han analizado 8 trabajos ${ }^{12,99,115,122-126}$, el último de ellos prospectivo y randomizado, que analizan la incidencia de hipertensión arterial con un tiempo de seguimiento de media superior a 14 años, en un grupo homogéneo de pacientes de ambos sexos. La eliminación del reflujo, bien de forma espontánea o por tratamiento quirúrgico no evita la predisposición a padecer hipertensión arterial en el futuro. La hipertensión aparece casi exclusivamente en presencia de cicatrices renales. Esto obliga en cierta manera a mantener un seguimiento a largo plazo de los pacientes con nefropatía por reflujo, especialmente en aquellos con lesiones renales difusas y bilaterales. El riesgo relativo de padecer hipertensión arterial en los pacientes con lesiones renales por cicatriz postpielonefrítica es del 2,92 (intervalo de confianza al 95\%: 1,2-7,132. La aparición de hipertensión arterial es independiente del grado de reflujo existente pre-tratamiento, de la persistencia del mismo y de la presencia de infecciones urinarias en el curso posterior al tratamiento quirúrgico. La aparición de hipertensión arterial severa se describe entre un 1,2 y $2,7 \%$ de las series ${ }^{125,127,128}$.

\section{DESARROLLO PONDOESTATURAL}

Los trabajos clásicos de la década de los 70 mostraban una tendencia de los niños con RVU a tener una talla y peso inferior al percetil correspondiente para su edad ${ }^{129}$ incluso reafirmados posteriormente por otros autores ${ }^{130}$ especialmente en los niños que padecían infecciones urinarias. Nuevamente se plantea la discusión sobre si alguno de los tratamientos escogidos para el RVU, médico o quirúrgico, modifican esta tendencia. Smellie ${ }^{131}$ no encuentra diferencias estadísticamente significativas en la velocidad de crecimiento y percentil en un grupo de 114 niñas sometidas a quimioprofilaxis con cotrimoxazol y nitrofurantoina frente a un grupo control. Merrell $^{132}$ y posteriormente Polito ${ }^{133}$ en un estudio retrospectivo sobre 94 pre-púberes, 30 de los cuales presentaban cicatrices renales (17bilaterales) en el DMSA, demostraba los beneficios del tratamiento quirúrgico. Para comparar las dos opciones de tratamiento entre si, se ha analizado cinco trabajos prospectivos. El trabajo prospectivo y randomizado entre tratamiento quirúrgico y médico realizado por Smellie y cols ${ }^{115}$, seguidos durante 10 años no encuentra diferencias estadísticamente significativas en este aspecto. Igualmente se han obtenido los mimos resultados en la rama europea del estudio internacional de reflujo tal y como ha descrito Olbing ${ }^{113}$ Otros estudios prospectivos anteriores a más corto plazo de seguimiento habían demostrado este mismo hecho ${ }^{85,134,135}$.

\section{MORBILIDAD DURANTE LA GESTACIÓN}

Conocemos la incidencia de bacteriuria durante la gestación (4-7\%) ${ }^{136,137}$. Además sabemos que las bacteriurias no tratadas durante la gestación sobrevienen en infección urinaria en un 20 a un $40 \%{ }^{137,138}$. Estas infecciones se han asociado a amenaza de parto pretérmino, prematuridad, y bajo peso al nacimiento ${ }^{139}$. Además de estos hechos las infecciones urinarias durante la gestación se han asociado a problemas severos 
para el recién nacido y la madre como la insuficiencia respiratoria en el recién nacido, la hipertensión arterial en la madre, la eclampsia, el shock séptico, la alteración de la función renal, la precipitación hacia insuficiencia renal en la madre, o la pérdida fetal. Las mujeres que han padecido infecciones urinarias durante la infancia, tienen un mayor riesgo de padecer bacteriurias asintomáticas durante la gestación entre el $37-50 \%{ }^{22,140,141}$. Si la paciente tiene cicatrices renales este riesgo se incrementa hasta el 47 $60 \%{ }^{142}$. El meta-análisis con 23.298 mujeres de 17 cohortes realizado por Romero ${ }^{143}$ demuestra que las pacientes con bacteriuria tienen un riesgo 2 veces superior de padecer parto pretérmino y 1,5 veces más riesgo de bajo peso al nacimiento del recién nacido que aquellas sin bacteriuria. Kindcaid-Smith ${ }^{144}$ demostró un riesgo 2,9 veces superior de pérdida de feto durante el segundo y tercer trimestre en las pacientes con bacteriuria y 1,8 veces más riesgo de padecer pre-eclampsia. En pacientes con nefropatía por reflujo y función renal reducida, las complicaciones durante el embarazo son importantes: mayor reducción de la función renal, parto pretérmino, toxemia y pérdida fetal. Las pacientes con insuficiencia renal en tratamiento con inhibidores de la enzima convertidora de angiotensina (IECA), tienen un especial riesgo de padecer oligoamnios ${ }^{145}$ Conocidas estas asociaciones cabe esperar igualmente que en una paciente embarazada con antecedentes de RVU y cicatrices renales la morbilidad gestacional, para madre e hijo estén aumentadas. La morbilidad durante el embarazo en pacientes afectas de reflujo ha sido estudiada en un grupo de pacientes con bacteriuria durante la gestación por Williams ${ }^{146}$. De su serie el $21 \%$ de las pacientes con bacteriuria presentaban RVU en una cistouretrografía realizada a los seis meses del parto, comparada con el 1,7\% de las pacientes sin bacteriuria escogidas aleatoriamente. Martinell ${ }^{22}$ encuentra en su serie un $37,5 \%$ de pielonefritis en pacientes embarazadas afectas de reflujo, independientemente de si tenían o no profilaxis antibiótica (33\% y 50\% respectivamente), frente a un $6 \%$ entre aquellas sin reflujo. Aunque estos datos hablan a favor de una mayor morbilidad durante el embarazo de las mujeres con RVU persistente, las series son escasas en número y no es posible obtener resultados con la suficiente evidencia científica. No se han encontrado trabajos prospectivos que comparen el tratamiento médico antibiótico profiláctico durante la gestación, aunque la mayoría de los autores son partidarios de su uso ${ }^{120,147}$. Sobre las pacientes intervenidas durante la infancia y posteriormente embarazadas se han encontrado artículos enfrentados en sus conclusiones. A nuestro juicio, la heterogeneidad de las series, en cuanto a grados de reflujo, lateralidad, y afectación renal por cicatrices, justifican estas discrepancias. Fryczkowski ${ }^{148}$ detecta hasta un $65 \%$ de bacteriurias en las mujeres embarazadas operadas por reflujo durante la infancia, comparadas con un $29 \%$ de bacteriurias entre las mujeres no embarazadas y también intervenidas. No comenta el porcentaje de pielonefritis del primer grupo durante la gestación. Austenfield y Snow ${ }^{149}$ encuentran un $57 \%$ de infecciones urinarias durante la gestación en aquellas pacientes intervenidas de RVU. Mansfield ${ }^{120}$ en un trabajo retrospectivo, compara dos grupos de pacientes: uno de intervenidas y otro de no intervenidas por reflujo, posteriormente embarazadas. En el grupo de las pacientes intervenidas el $65 \%$ desarrollaron infección urinaria frente al $15 \%$ de las no intervenidas, siendo estadísticamente significativo este dato. Sin embargo no hubo diferencias significativas en cuanto al porcentaje de pielonefritis $(5 \%$ frente a $1,5 \%$, operadas vs. no operadas) ni en abortos espontáneos (21/141 [15\%] vs. $14 / 75$ [18\%]). Bukowski ${ }^{147}$ no encuentra diferencias significativas en su serie con respecto al desarrollo de pielonefritis durante el embarazo entre las pacientes intervenidas por reflujo $(6,5 \%)$, que las intervenidas no embarazadas $(6,2 \%)$. El porcentaje de abortos espontáneos fue en esta serie del $17 \%$ y no se encontró relación entre las pielonefritis y la pérdida fetal. Muy interesante nos parece el estudio de McGladdeery ${ }^{141}$ que compara mujeres con historia de infección urinaria durante la infancia con y sin cicatrices renales, incluyendo aquellas con historia de RVU en cada grupo. Las pacientes con RVU y cicatrices renales tuvieron un riesgo de padecer hipertensión y eclampsia 3 y 7 veces superior al resto de los grupos, respectivamente. Las pacientes sin cicatrices renales conocidas y con reflujo tuvie- 
ron un ligero incremento del riesgo de padecer hipertensión durante el embarazo, en el tercer trimestre, pero esto no supuso un aumento significativo del riesgo de padecer infección urinaria.

\section{COMPLICACIONES ASOCIADAS AL TRATAMIENTO}

\section{Obstrucción ureteral}

Esta complicación es la más frecuentemente descrita entre los textos seleccionados para esta revisión. Se han encontrado 51 artículos en los que se describe dicha complicación, 35 correspondientes a reimplantes con cirugía abierta, transvesical y extravesical ${ }^{81,87,100,101,150,180}$ y 18 correspondientes a tratamientos endoscópicos, utilizando gran variedad de sustancias para la inyección, ${ }^{38,39,46,47,49,56,58,59,181-187}$. El índice de obstrucciones ureterales post-reimplante se encuentra entre el 0,9 y el $2 \%$, siendo las series anteriores a 1986 las que presentan una mayor tasa de obstrucción, tal y como se ha descrito en la revisión realizada por el grupo de expertos de la Asociación Americana de Urología ${ }^{32}$. No se han encontrado diferencias significativas con respecto a la técnica quirúrgica practicada, tanto transvesical como extravesicalmente. La tasa de reoperaciones requerida para solucionar esta obstrucción ha variado igualmente del 0,3 al 9,1\%, lo que supone una incidencia media de reintervenciones por obstrucción tras reimplante ureteral del $2 \%$.

De los 489 uréteres tratados mediante inyección con polidimetilsiloxano ${ }^{38,44-46,52,60,188}$, sólo en un caso de la serie de Al-Hunayan, se produjo obstrucción post-inyección siendo necesario el reimplante. En el estudio más amplio que conocemos de Puri ${ }^{49}$, sobre un total de 53 urólogos de 41 centros diferentes así como en otro estudio del mismo autor ${ }^{189} 3$ años antes, en el ámbito europeo, sobre un total de 8.332 niños tratados con diferentes grados de RVU (I: 3,3\%, II: $31,2 \%$, III: $42,5 \%$, IV: $18,1 \%$ y V:4,7\%), con una tasa de éxito global del $89 \%$ se describieron 41 obstrucciones ureterales post-inyección $(0,33 \%)$ que requirieron reimplante ureteral. Stenberg ${ }^{190}$ en su serie utiliza las microesferas de dextrómero de ácido hialurónico sódico (Deflux system ${ }^{\circledR}$ ) y no encuentra casos de obstrucción ureteral postinyección.

\section{Hemorragia}

No se han encontrado diferencias significativas entre las diferentes técnicas quirúrgicas utilizadas en cirugía abierta siendo los casos de sangrado (hemorragia o hematoma) del $0,26 \%, 1,2 \%$. No se ha encontrado el sangrado como complicación en ninguno de los trabajos seleccionados y revisados sobre tratamiento endoscópico.

\section{Infección herida quirúrgica}

La tasa de infección de herida quirúrgica, tras reimplante ureteral, ha sido descrita entre el 0,8 y el $1,2 \%{ }^{179,191}$. No se han encontrado diferencias significativas entre los procedimientos transvesicales y extravesicales.

\section{Alteraciones funcionales postoperatorias (lesión vesical)}

Las alteraciones miccionales tras el reimplante ureteral han sido descritas no sólo desde el punto de vista clínico, sino incluso con modelos anatómicos que demuestran la posible afectación del plexo hipogástrico situado cercano a la unión urétero-vesical y que puede ser lesionado durante la desinserción ureteral ${ }^{192}$ Especialmente este hecho se ha descrito en los reimplantes ureterales extravesicales ${ }^{193}$ en la que se produce una sección longitudinal del detrusor vesical, y en concreto en los pacientes que presentaban antecedentes de trastornos funcionales, o en los que se realiza cirugía bilateral en un solo tiempo quirúrgico. Para la cirugía extravesical tipo LichGregoir se ha descrito una incidencia de alteraciones funcionales significativas, que requirieron tratamiento en un 6,5\% ${ }^{194}$. En esta serie un 2,5\% fueron tratados con anticolinérgicos y un $4 \%$ con cateterismos vesicales o sondaje permanente entre 2 y 21 días. Las alteraciones funcionales en los pacientes tratados mediante inyección endoscópica han sido analizadas en la mayoría de los trabajos como causa de fallo de la técnica y no como consecuencia de la misma. Cappozza ${ }^{63}$ describe un $4,3 \%$ de pacientes que presentaban disfunciones miccionales no conocidas previamente tras la inyección endoscópica que no requirieron tratamiento postoperatorio. Llama la atención como ninguna de las series revisadas basa en estudios urodinámicos las hiperactividad vesical descritas en sus series, que en la totalidad de los 
casos son definidas como alteraciones clínicas en el patrón miccional, con cuadros de urgencia miccional y urgencia - incontinencia. Serían necesarios más estudios en este sentido que pudiesen aportar evidencias significativas en cuanto a la aparición de alteraciones funcionales, pues posiblemente estén infravaloradas. No hemos encontrado así mismo ningún trabajo que compare de manera randomizada esta complicación entre el tratamiento quirúrgico abierto y el endoscópico.

\section{Aparición de RVU contralateral}

Este hecho ha sido sobradamente descrito en casos de aparición de RVU contralateral "de novo", así como de reaparición de un RVU contralateral solucionado espontáneamente ${ }^{195}$ Se ha aludido a la existencia de un mecanismo de contra-presión, denominado "pop-off" que explicaría la aparición de un RVU de novo cuando la presión intravesical aumentase al suprimirse el RVU conocido. Este hecho no ha podido demostrarse tras procedimientos endoscópicos en RVU de alto grado ${ }^{196}$ No se ha encontrado relación entre la aparición de reflujo contralateral y la edad, o grado de reflujo ${ }^{197}$. Se ha descrito igualmente la aparición de RVU ipsilateral en el caso de duplicidades completas, aunque la mayoría de ellas no han necesitado de tratamiento quirúrgico para su resolución, siendo ésta espontánea196,198. Elder y cols. han determinado el porcentaje estimado de RVU contralateral para diferentes grados de RVU intervenido $(1,52 \%$ para el grado I y II, $2,8 \%$ para el grado III, 3,66\% para el grado IV y 2,53 para el grado V) y diferentes técnicas quirúrgicas empleadas, no encontrando tampoco en éstas, diferencias estadísticamente significativas. No se encontraron diferencias significativas con respecto al tratamiento endoscópico por si sólo ni analizando el grado de reflujo preoperatorio o el material usado para la inyección.

\section{Dolor postoperatorio}

Se trata de uno de los efectos indeseables más difícilmente cuantificables a pesar de que se han utilizado varias escalas de dolor, aplicadas al control analgésico postoperatorio en niños ${ }^{467}$. Se han descrito diferentes pautas de tratamiento como el bloqueo caudal con un periodo efectivo de analgesia de 12 horas postinyección sin presentar complicaciones relacionadas con el procedimiento, el uso de catéteres epidurales con analgesia continua, que se mantienen durante el periodo de cateterización vesical, disminuyendo la cantidad de analgésicos administrados durante el postoperatorio, pero con una incidencia mayor de episodios febriles postquirúrgicos, una estancia media similar a los analgésicos parenterales habituales y un mayor número de complicaciones asociadas al propio catéter epidural ${ }^{199}$. También se ha descrito el uso de ketokerolato intravenoso como tratamiento analgésico para controlar los espasmos vesicales derivados del uso del catéter vesical, estableciéndose su seguridad y efectividad por encima del tratamiento placebo ${ }^{200}$ Por último se ha utilizado la analgesia intravesical con mórficos, que igualmente reduce los espasmos vesicales tan mal tolerados por el niño. Sin embargo ElGhoneimi ${ }^{201}$ demuestra en un estudio controlado y randomizado que no existen diferencias significativas entre este y el placebo. No hemos encontrado datos objetivos que avalen la teoría de que el reimplante extavesical o la cirugía laparoscópica disminuyen el dolor postoperatorio comparado con el resto de procedimientos quirúrgicos.

\section{Días de hospitalización postoperatoria}

Se han analizado 10 estudios $^{151,157,163,164,167}$, 168,202-205 en los que de forma principal ha sido analizado este parámetro, sobre un total de 637 pacientes, todos ellos realizados con cirugía abierta de reimplante ureteral. La estancia media osciló entre 2,4 días y 13,9 días aunque parece variar en función de la técnica usada (menor para las técnicas exravesicales que para las de reimplante intravesical) y de si se dejan catéteres ureterales o no (5,4-5,6 días frente a los 2,7-4,2 días respectivamente). Se han podido analizar varios trabajos en los que se realiza de forma ambulatoria o con ingresos inferiores a 24 horas de hospitalización el tratamiento endoscópico del reflujo, con diversas sustancias ${ }^{40,41,45,46,49,52,107,206,207 .}$

\section{Complicaciones asociadas al uso de los} materiales para el tratamiento endoscópico

Las complicaciones de los diferentes materiales 
usados para el tratamiento endoscópico han sido analizadas en la Tabla 6, tanto para aquellos en los que está aprobado el uso en humanos como los usados sólo a nivel experimental.

\section{EFECTOS INDESEABLES DEL TRATAMIENTO MÉDICO}

Las complicaciones derivadas directamente del manejo médico se refieren especialmente a

\section{Tabla 6}

Relación de materiales utilizados para el tratamiento endoscópico del RVU tanto en humanos como en experimentación animal, referencias, complicaciones relacionadas con su uso y resultados globales de las series citadas

\begin{tabular}{|c|c|c|c|c|}
\hline \multicolumn{5}{|c|}{ Sustancias utilizadas para la inyección submeática en el tratamiento del RVU } \\
\hline Sustancia & Composición & Referencias & Efectos Secundarios & Éxito \\
\hline \multicolumn{5}{|l|}{ Materiales Autólogos } \\
\hline Condrocitos & $\begin{array}{l}\text { Obtenidos de ingeniería } \\
\text { tisular }\end{array}$ & {$[244,245]$} & No se han observado. & $\begin{array}{l}79 \%{ }^{246} . \text { Faltan resultados a } \\
\text { largo plazo }\end{array}$ \\
\hline Células musculares & $\begin{array}{l}\text { Microesferas biodegradables } \\
\text { con células musculares en } \\
\text { su interior }\end{array}$ & {$[247,248]$} & No se han observado & Uso experimental \\
\hline Grasa autóloga & & {$[249,250]$} & No se han observado & Menores al $17 \%{ }^{251}$ \\
\hline Colágeno autólolgo & & [252] & No se han observado. & Sólo uso experimental animal \\
\hline \multicolumn{5}{|l|}{ Materiales Heterólogos } \\
\hline $\begin{array}{l}\text { Coaptite }{ }^{\circledR} \text { Bioform, } \\
\text { Franksuille, WI. }\end{array}$ & $\begin{array}{l}\text { Hidroxiloapatita } \\
\text { Ca10(PO4)6(OH)2 }\end{array}$ & $\begin{array}{l}\text { No existen referencias } \\
\text { sobre su uso en el } \\
\text { tratamiento del RVU, } \\
\text { sólo en incontinencia } \\
\text { urinaria de esfuerzo }\end{array}$ & $\begin{array}{l}\text { No se han observado } \\
\text { en el tratamiento del } \\
\text { RVU }\end{array}$ & \\
\hline Teflon ${ }^{\circledR}$ & Politetrafluoruro de etileno & {$[59,189,253-261]$} & $\begin{array}{l}\text { Migración de la sustancia } \\
\text { local y a distancia a } \\
\text { pulmón y cerebro. } \\
\text { Determinación experimental } \\
\text { animal en el caso de RVU, } \\
\text { aunque demostrado en } \\
\text { humanos tras su uso en } \\
\text { incontinencia urinaria }{ }^{262-264}\end{array}$ & $92 \%$ \\
\hline $\begin{array}{l}\text { Colágeno bovino: } \\
\text { Cotigen }{ }^{\circledR} \\
\text { Zyderm }{ }^{\circledR} \\
\text { Zyplast }{ }^{\circledR} \\
\text { Bard corporation, } \\
\text { Atlanta, Ge. } \\
\text { Collagen Corporation, } \\
\text { Palo Alto, Ca. }\end{array}$ & $\begin{array}{l}\text { Colágeno bovino entrelazado } \\
\text { con glutaraldehído } \\
\text { biodegradable (Colágeno GAX) }\end{array}$ & {$[246,265,266,267]$} & $\begin{array}{l}\text { Hasta la fecha no se han descrito } \\
\text { fenómenos de migración y la } \\
\text { reacción inflamatoria es leve } \\
\text { sin formación de granulomas } \\
\text { Sin embargo se han descrito } \\
\text { reacciones inmunitarias adversas, } \\
\text { [269]hipersensibilidad aguda, } \\
\text { enfermedades del tejido conectivo, } \\
\text { lupus eritematoso, polimiositis y } \\
\text { dermatosis }^{270}\end{array}$ & $\begin{array}{l}62 \% \text {, con descenso al } 58 \% \text { a los } \\
3 \text { años. Estudio prospectivo: }{ }^{271}\end{array}$ \\
\hline Macroplastico® & Polidimetilxilosano & $\begin{array}{l}{[44-46,60,181} \\
272,273]\end{array}$ & $\begin{array}{l}\text { Migración de la sustancia, } \\
\text { experimentación } \\
\text { animal }^{274,275} \text {. }\end{array}$ & $90 \%$ \\
\hline $\begin{array}{l}\text { Deflux }{ }^{\circledR} \text { (Deflux, } \\
\text { Q-Med AB, Upsala, } \\
\text { Sweden) }\end{array}$ & $\begin{array}{l}\text { Microesferas de dextranómero } \\
\text { suspendidas en solución } \\
\text { de hialuronato sódico. }\end{array}$ & {$[52,190,256]$} & $\begin{array}{l}\text { Las microesferas no son } \\
\text { inmunogénicas ni producen } \\
\text { migración. }\end{array}$ & $\begin{array}{l}70 \% \text {. Faltan estudios a largo } \\
\text { plazo, dado que el producto se } \\
\text { reabsorve. }\end{array}$ \\
\hline $\begin{array}{l}\text { Sistema de balón } \\
\text { autodesechable }\end{array}$ & $\begin{array}{l}\text { Hidroximetil acrilato } \\
\text { Poli-N-vinil pirrolidonona }\end{array}$ & {$[276]$} & No se han observado & $\begin{array}{l}\text { Utilizado solo en ensayos } \\
\text { animales y ensayos clínicos } \\
\text { en fase II para RVU. }\end{array}$ \\
\hline Ivalon & Alcohol polivinílico & [277] & $\begin{array}{l}\text { En experimentación animal, } \\
\text { resulta carcinogénico }\end{array}$ & \\
\hline
\end{tabular}


aquellas relacionadas con los estudios radiológicos, tanto convencionales como isotópicos, es decir: radiaciones ionizantes, reacciones alérgicas al contraste yodado y cateterismos uretrales.

\section{Radiaciones ionizantes}

La Comisión Europea y el Ministerio de Sanidad y Consumo nos recuerdan a través de la guía de indicaciones para la correcta solicitud de pruebas de diagnóstico por la imagen ${ }^{208}$ que aunque las ventajas de la realización de este tipo de pruebas compensan con creces los leves riesgos de la radiación, ni siquiera las pequeñas dosis están totalmente exentas de riesgos. Las dosis efectivas características de algunas técnicas habituales en radiodiagnóstico se especifican en la Tabla 7 destacando especialmente aquellas solicitadas en el estudio o seguimiento de un RVU.

La cistografía isotópica directa es realizada con Tc99m en cloruro sódico al 0,9\% en un medio estéril y apirógeno. No se conocen contraindicaciones asociadas al propio isótopo y no hemos encontrado estudios en animales que demuestren su potencial carcinogénico o alteraciones en la fertilidad.

Las dosis recomendadas para el estudio de RVU son de 18,5 a $37 \mathrm{MBq}(0,5-1 \mathrm{mCi})$, siendo las dosis de radiación absorbidas en niños. No se han descrito reacciones anafilácticas con el uso de la cistografía isotópica.

\section{Tabla 7}

Dosis efectivas características en radiodiagnóstico en la década de los años noventa

\begin{tabular}{lccc}
\hline $\begin{array}{l}\text { Procedimiento } \\
\text { diagnóstico }\end{array}$ & $\begin{array}{c}\text { Dosis efectiva } \\
\text { característica } \\
(\mathrm{mSv})\end{array}$ & $\begin{array}{c}\text { Número equivalente } \\
\text { de radiografías } \\
\text { de tórax }\end{array}$ & $\begin{array}{c}\text { Período equivalente } \\
\text { aproximado de } \\
\text { radicación natural } \\
\text { de fondo }\end{array}$
\end{tabular}

\section{Radiografías}

Tórax

Columna dorsal

Columna lumbar

Cadera

Pelvis

Abdomen

UIV

TC de abdomen

o pelvis

0,002
0,7
1,3
0,3
0,7
1,0
2,5
10

1
35
65
15
35
50
125
500

\section{Gammagrafía}

Renal

1

50

3 días

4 meses

7 meses

7 semanas

4 meses

6 meses

14 semanas

4,5 años

Para realizar las cistografias isotópicas directas y convencionales es necesario someter al paciente a cateterismo transuretral del cual se derivan complicaciones determinadas en un 35\% de los casos, en forma de sintomatología miccional irritativa e infecticos), ésta se hace imprescindible para evitar la infección urinaria. No se han encontrado documenque recojan las alteraciones psicológicas en los reacciones alérgicas derivadas del uso de contraste yodado son infrecuentes en la población infantil, un 6\% consideradas menores (náuseas, vómitos, urtipero sin haberse descrito muertes por reacciones

\section{Reacciones adversas medicamentosas}

La quimioprofilaxis antibiótica que se utiliza en el tratamiento médico continuado se administra a razón de 1/4 o 1/3 de la dosis terapéutica completa. Los fármacos habitualmente utilizados y recogidos en la literatura científica son: el trimetopim, el cotrimoxazol (sulfametoxazoltrimetopim), la nitrofurantoína, y las cefalosporinas de primera y segunda generación por vía oral (cefonicid, cefalexina y cefazolina). Las bajas dosis administradas y la comprobada seguridad del fármaco en niños producen muy bajos indices de efectos secundarios y son escasamente recogidos en la literatura. De una parte estos efectos secundarios son habitualmente menores (mal sabor de boca). De otra parte muchos de ellos son difícilmente achacables únicamente al fármaco como 
dolores abdominales inespecíficos y difusos. No es frecuente encontrar referidos en los trabajos de tratamiento médico abandonos de la medicación por estos efectos, y la mayoría de los graves efectos secundarios se describen en adultos y en tratamientos a dosis plenas. En cualquier caso la prolongación en el tiempo del tratamiento antibiótico es una inquietud habitualmente referida por los padres que puede condicionar el tratamiento a seguir. Los fármacos anticolinérgicos utilizados también en el tratamiento médico del reflujo asociado a inestabilidad vesical son el cloruro de trospio, la oxibutinina y la tolterodina. La fiabilidad de los tres ha sido probadamente comprobada tanto en adultos como en niños. Merece la pena destacar que, en nuestra experiencia, y como se recoge en la literatura la tolerabilidad del fármaco es mucho mayor en los niños que en los adultos. Al igual que el tratamiento antibiótico, la mayoría de los artículos revisados hacen referencia a efectos secundarios aparecidos en los adultos, en especial con la tolterodina, fármaco que en un principio no fue evaluado en la edad infantil, y del que ya se tienen evidencias sobre su seguridad y efectividad en los niños ${ }^{213,214}$.

\section{CONCLUSIONES DE LA REVISIÓN}

Tanto el tratamiento médico como el quirúrgico presentan la misma efectividad en la resolución de los grados I, II y III de RVU, recomendándose el primero de ellos como tratamiento inicial tras el diagnóstico. Grado de recomendación A.

El tratamiento endoscópico es igual de efectivo que la cirugía abierta para los grados I, II y III. Grado de recomendación A.

La incidencia de efectos indeseables secundarios al tratamiento endoscópico es menor que la cirugía abierta. Grado de recomendación A.

Para los grados I, II y III, el tratamiento quirúrgico recomendado es la inyección endoscópica, no existiendo diferencias en cuanto a la efectividad de las distintas sustancias. Grado de recomendación $\mathrm{B}$.

Para los grados IV y V no existen evidencias suficientes que aconsejen o desaconsejen cualquiera de los tratamientos. Grado de recomendación $\mathrm{C}$.

El tratamiento quirúrgico abierto, para cualquier grado de RVU sólo presenta superioridad con respecto al tratamiento médico en el número de episodios de pielonefritis aguda durante el seguimiento. No existen diferencias entre ambos para: las infecciones urinarias, la hipertensión arterial, la insuficiencia renal, el crecimiento renal, el crecimiento del niño ni la morbilidad gestacional. Grado de recomendación A.

Esta misma conclusión no es posible aplicarla con los datos publicados para el tratamiento endoscópico. Grado de recomendación C.

\section{COMPARACIÓN CON EL TRABAJ O APARECIDO EN THE COCHRANE LIBRARY 288}

Las dos revisiones efectuadas en época cercana alcanzan las mismas conclusiones con respecto al tratamiento del RVUP sin poder dar respuesta concreta a diversas interrogantes que permitan protocolizar con evidencia científica el tratamiento exacto para cada grado de reflujo y situación clínica, debiendo utilizar recomendaciones sin base firme para la toma de decisiones.

Tal y como Wheeler y cols. destacan en su trabajo, consideramos imprescindible la creación de ensayos clínicos, prospectivos y randomizados que permitan con la suficiente evidencia científica recomendar la adopción o la no adopción de las distintas opciones terapéuticas para evitar la lesión renal significativa y progresiva.

Esta revisión sistemática fue defendida como Tesis Doctoral el 20 de enero de 2004 en la Universidad Complutense de Madrid y calificada con sobresaliente "cum laude". Fue dirigida por el Prof. Angel Silmi Moyano.

\section{Agradecimientos}

El mayor de los agradecimientos al Dr. José $M^{\mathbf{a}}$ Garat, mentor de este trabajo y referente de la urología pediátrica.

\section{REFERENCIAS}

1. Eisenberg J. Ten lessons of evidence-based technology assessment. JAMA, 1999. 282: 1865-1869.

2. Mulrow. Rationale for systematic reviews. BMJ, 1994. 309: 597-599.

3. Jiménez F, Meta-análisis en investigación clínica en urologia. Arch Esp Urol, 2003. 56(6):657-665.

4. Bourchier D, G.D. Abbott, and T.M. Maling. Radiological abnormalities in infants with urinary tract infections. Arch Dis Child, 1984. 59(7):620-624.

5. Jodal U. and U. Lindberg, Guidelines for management of children with urinary tract infection and vesico-ureteric reflux. Recommendations from a Swedish state-of-the-art conference. Swedish Medical Research Council. Acta Paediatr Suppl, 1999. 88(431): 87-89. 
6. Jodal U, O. Koskimies, E Hanson, GLohr, H Olbing, Smellie, and T. Tamminen-Mobius, Infection pattern in children with vesicoureteral reflux randomly allocated to operation or long-term antibacterial prophylaxis. The International Reflux Study in Children. J Urol, 1992. 148(5 Pt 2):1650-1652.

7. Jodal U, S. Hansson, and K. Hjalmas. Medical or surgical management for children with vesico-ureteric reflux? Acta Paediatr Suppl, 1999. 88(431):53-61.

8. Marild S. and U. Jodal, Incidence rate of first-time symptomatic urinary tract infection in children under 6 years of age. Acta Paediatr, 1998. 87: 549-552.

9. Krober, M.S., J.W. Bass, J.M. Powell, F.R. Smith, and D.S.Y. Seto, Bacterial and viral pathogens causing fever infants less than 3 months old. Am J Dis Child, 1985. 139: 889-92.

10. Hobert, K., S.M. Chao, D.M. Keller, C.J. Hixson, and G. Ellis, Prevalence of urinary tract infection in febrile infants. J Pediatr, 1993. 123: 17-23.

11. Smellie, J.M., I.C. Normand, and G. Katz, Children with urinary infection: a comparison of those with and those without vesicoureteric reflux. Kidney Int, 1981. 20(6): 717 722 .

12. Smellie, J.M., N.P. Prescod, P.J. Shaw, R.A. Risdon, and T.N. Bryant, Childhood reflux and urinary infection: a follow-up of 10-41 years in 226 adults. Pediatr Nephrol, 1998. 12(9): 727-736.

13. Committee., I.R.S., Medical versus surgical treatment of primary vesicoureteral reflux. Pediatrics, 1981. 67:392400 .

14. Anderson, N.G., G.D. Abbott, N. Mogridge, R.B. Allan, T.M. Maling, and J.E. Wells, Vesicoureteric reflux in the newborn: relationship to fetal renal pelvic diameter. Pediatr Nephrol, 1997. 11(5): 610-616.

15. Zerin, J.M., M.L. Ritchey, and A.C. Chang, Incidental vesicoureteral reflux in neonates with antenatally detected hydronephrosis and other renal abnormalities. Radiology, 1993. 187(1):157-60.

16. Clautice-Engle, T., N.G. Anderson, and R.B. Allan, Diagnosis of obstructive hydronephrosis in infants.comparasion sonograms performed 6 days and 6 weeks afetr birth. AJR Am J Roentgenol, 1995. 164:. 963-967.

17. Dudley, J.A., J.M. Haworth, M.E. McGraw, J.D. Frank, and E.J. Tizard, Clinical relevance and implications of antenatal hydronephrosis. Arch Dis Child Fetal Neonatal Ed, 1997. 76(1):. F31-4.

18. Pal, C.R., J.R. Tuson, D.R. Lindsell, K. McHugh, P.L. Hope, and K. Ives, The role of micturating cystourethrography in antenatally detected mild hydronephrosis. Pediatr Radiol, 1998. 28(3): 152-5.

19. Walsh, G. and P.A. Dubbins, Antenatal renal pelvis dilatation: a predictor of vesicoureteral reflux? AJR Am J Roentgenol, 1996. 167(4): 897-900.

20. Atala, A., P. Ellsworth, J. Share, H. Paltiel, R.D. Walker, and A.B. Retik, Comparison of sonicated albumin enhanced sonography to fluoroscopic and radionuclide voiding cystography for detecting vesicoureteral reflux. J Urol, 1998. 160(5): 1820-2.

21. Riccabona, M., C.J. Mache, and F. Lindbichler, Echoenhanced color Doppler cystosonography of vesicoureteral reflux in children. Improvement by stimulated acoustic emission. Acta Radiol, 2003. 44(1): 18-23.

22. Martinell, J., U. Jodal, and G. Lidin-Janson, Pregnancies in women with and without renal scarring after urinary infections in childhood. Bmj, 1990. 300(6728): 840-4.
23. Polito, C., A. La Manna, L. Mansi, P.F. Rambaldi, M.R. Papale, A. Marte, and R. Di Toro, Body growth in early diagnosed vesicoureteric reflux. Pediatr Nephrol, 1999. 13(9): 876-9.

24. Skoog, S.J., A.B. Belman, and M. Majd, A nonsurgical approach to the management of primary vesicoureteral reflux. J Urol, 1987. 138(4 Pt 2): 941-6.

25. Skoog, S.J. and A.B. Belman, Primary vesicoureteral reflux in the black child. Pediatrics, 1991. 87(4): 538-43.

26. Duckett, J.W., Vesicoureteral reflux: a 'conservative' analysis. Am J Kidney Dis, 1983. 3(2): 139-44.

27. Goldraich, N.P. and I.H. Goldraich, Followup of conservatively treated children with high and low grade vesicoureteral reflux: a prospective study. J Urol, 1992. 148(5 Pt 2): 1688-92.

28. Lenaghan, D., J.G. Whitaker, F. Jensen, and F.D. Stephens, The natural history of reflux and long-term effects of reflux on the kidney. J Urol, 1976. 115(6): 728-30.

29. Arant, B.S., Jr., Medical management of mild and moderate vesicoureteral reflux: followup studies of infants and young children. A preliminary report of the Southwest Pediatric Nephrology Study Group. J Urol, 1992. 148(5 Pt 2): $1683-7$.

30. Belman, A.B. and S.J. Skoog, Nonsurgical approach to the management of vesicoureteral reflux in children. Pediatr Infect Dis J, 1989. 8(8): 556-9.

31. Tamminen-Mobius, T., E. Brunier, K.D. Ebel, R. Lebowitz, H. Olbing, U. Seppanen, and R. Sixt, Cessation of vesicoureteral reflux for 5 years in infants and children allocated to medical treatment. The International Reflux Study in Children. J Urol, 1992. 148(5 Pt 2): 1662-6.

32. Elder, J.S., C.A. Peters, B.S. Arant, Jr., D.H. Ewalt, C.E. Hawtrey, R.S. Hurwitz, T.S. Parrott, H.M. Snyder, 3rd, R.A. Weiss, S.H. Woolf, and V. Hasselblad, Pediatric Vesicoureteral Reflux Guidelines Panel summary report on the management of primary vesicoureteral reflux in children. J Urol, 1997. 157(5): 1846-51.

33. Solok, V., A. Erozenci, A. Kural, and A. Oner, Correction of vesicoureteral reflux by the Gil-Vernet procedure. Eur Urol, 1988. 14(3): 214-5.

34. Glassberg, K.I., G. Laungani, R.J. Wasnick, and K. Waterhouse, Transverse ureteral advancement technique of ureteroneocystostomy (Cohen reimplant) and a modification for difficult cases (experience with 121 ureters). J Urol, 1985. 134(2): 304-7.

35. Starr, A., Ureteral plication: a new concept in ureteraltapering for megaureter. Invest Urol, 1979. 17: 153.

36. Kalicinski, Z., J. Kansy, and B. Kotarbinska, Surgery of megaureters:modification of Hendren's operation. J Pediatr Surg, 1977. 12: 183.

37. Hendren, W.H., Operative repair of megaureter in children. J Urol, 1969. 101: 491.

38. Serrano Durbá, A., F. Estornell Moragues, C. Dominguez Hinarejos, M. Martinez Verduch, and F. Garcia Ibarra, Eficacia del tratamiento endoscópico del reflujo vesicoureteral primario en la infancia con polidimetilxilosano. Urol Int Invest, 2002. 7(4):. 490-493.

39. Farkas, A., E.Z. Moriel, and S. Lupa, Endoscopic correction of vesicoureteral reflux: our experience with 115 ureters. J Urol, 1990. 144(2 Pt 2): 534-6; discussion 545.

40. Chertin, B., E. Colhoun, M. Velayudham, and P. Puri, Endoscopic treatment of vesicoureteral reflux: 11 to 17 years of followup. J Urol, 2002. 167(3): 1443-5; discussion 1445-6. 
41. Chertin, B., D. De Caluwe, and P. Puri, Endoscopic treatment of primary grades IV and $\mathrm{V}$ vesicoureteral reflux in children with subureteral injection of polytetrafluoroethylene. J Urol, 2003. 169(5): 1847-9; discussion 1849.

42. Stenberg, A., E. Larsson, and G. Lackgren, Endoscopic treatment with dextranomer-hyaluronic acid for vesicoureteral reflux: histological findings. J Urol, 2003. 169(3): 1109-13.

43. Brown, S., Open versus endoscopic surgery in the treatment of vesicoureteral reflux. J Urol, 1989. 142(2 Pt 2): 499-500; discussion 501.

44. Aboutaleb, H., S. Bolduc, J. Upadhyay, W. Farhat, D.J. Bagli, and A.E. Khoury, Subureteral polydimethylsiloxane injection versus extravesical reimplantation for primary low grade vesicoureteral reflux in children: a comparative study. J Urol, 2003. 169(1): 313-6.

45. Herz, D., A. Hafez, D. Bagli, G. Capolicchio, G. McLorie and A. Khoury, Efficacy of endoscopic subureteral polydimethylsiloxane injection for treatment of vesicoureteral reflux in children: a North American clinical report. J Urol, 2001. 166(5): 1880-6.

46. Al-Hunayan, A.A., E.O. Kehinde, M.A. Elsalam, and R.S. Al-Mukhtar, Outcome of endoscopic treatment for vesicoureteral reflux in children using polydimethylsiloxane. $\mathrm{J}$ Urol, 2002. 168(5): 2181-3.

47. Lipsky, H. and E. Wurnschimmel, Endoscopic treatment of vesicoureteric reflux with collagen. Five years' experience. Br J Urol, 1993. 72(6): 965-8.

48. Capozza, N. and P. Caione, Dextranomer/hyaluronic acid copolymer implantation for vesico-ureteral reflux: a randomized comparison with antibiotic prophylaxis. J Pediatr, 2002. 140(2): 230-4

49. Puri, P. and C. Granata, Multicenter survey of endoscopic treatment of vesicoureteral reflux using polytetrafluoroethylene. J Urol, 1998. 160(3 Pt 2): 1007-11; discussion 1038.

50. Haferkamp, A., K. Mohring, G. Staehler, H.J. Gerner, and J. Dorsam, Long-term efficacy of subureteral collagen injection for endoscopic treatment of vesicoureteral reflux in neurogenic bladder cases. J Urol, 2000. 163(1): 274-7.

51. Caldamone, A.A. and D.A. Diamond, Long-term results of the endoscopic correction of vesicoureteral reflux in children using autologous chondrocytes. J Urol, 2001. 165(6 Pt 2): 2224-7.

52. Oswald, J., M. Riccabona, L. Lusuardi, G. Bartsch, and C. Radmayr, Prospective comparison and 1-year follow-up of a single endoscopic subureteral polydimethylsiloxane versus dextranomer/hyaluronic acid copolymer injection for treatment of vesicoureteral reflux in children. Urology, 2002. 60(5): 894-7; discussion 898.

53. Vereecken, R.L. and W. Proesmans, Results of endoscopic treatment for vesico-ureteric reflux. Eur Urol, 1995. 27(1): 76-9.

54. Lacombe, A., Ureterovesical reimplantation after failure of endoscopic treatment of reflux by submucosal injection of polytef paste. Eur Urol, 1990. 17(4): 318-20.

55. Dodat, H. and P. Takvorian, Treatment of vesicoureteral reflux in children by endoscopic injection of Teflon. Review of 2 years of experience. Eur Urol, 1990. 17(4): 304-6.

56. Sauvage, P., S. Geiss, C. Saussine, S. Laustriat, F. Becmeur, J. Bientz, D. Christmann, E. Roy, and L. Marcellin, Analysis and perspectives of endoscopic treatment of vesicoureteral reflux in children with a 20-month follow-up. Eur Urol, 1990. 17(4): 310-3.
57. Schulman, C.C., D. Pamart, M. Hall, F. Janssen, and F.E. Avni, Vesicoureteral reflux in children: endoscopic treatment. Eur Urol, 1990. 17(4): 314-7.

58. Davies, N. and J.D. Atwell, Primary vesicoureteric reflux: treatment with subureteric injection of Polytef paste. $\mathrm{Br} \mathrm{J}$ Urol, 1991. 67(5): 536-40.

59. Bhatti, H.A., H. Khattak, and V.E. Boston, Efficacy and causes of failure of endoscopic subureteric injection of Teflon in the treatment of primary vesicoureteric reflux. $\mathrm{Br}$ J Urol, 1993. 71(2): 221-5.

60. Ozyavuz, R., G.K. Ozgur, and A.K. Yuzuncu, Subureteric polydimethylsiloxane injection in the treatment of vesicoureteric reflux. Int Urol Nephrol, 1998. 30(2): 123-6.

61. Reunanen, M., Endoscopic collagen injection: its limits in correcting vesico-ureteral reflux in duplicated ureters. Eur Urol, 1997. 31(2): 243-5.

62. O'Donnell, B. and P. Puri, Treatment of vesicoureteric reflux by endoscopic injection of Teflon. Br Med J (Clin Res Ed), 1984. 289(6436): 7-9.

63. Capozza, N., A. Lais, E. Matarazzo, S. Nappo, M. Patricolo, and P. Caione, Influence of voiding dysfunction on the outcome of endoscopic treatment for vesicoureteral reflux. J Urol, 2002. 168(4 Pt 2): 1695-8.

64. Lackgren, G., N. Wahlin, E. Skoldenberg, and A. Stenberg, Long-term followup of children treated with dextranomer/hyaluronic acid copolymer for vesicoureteral reflux. J Urol, 2001. 166(5): 1887-92.

65. Pylkkänen, J., J. Vilska, and O. Koskimies, The value of level diagnosis of childhood urinary tract infection in predicting renal injury. Acta Paediatr Scand, 1981. 70: 87983.

66. Linne, T., O. Fituri, R. Escobar-Billing, A. Karlsson, I. Wikstad, A. Aperia, and K. Tullus, Functional parameters and 99m-technetium dimercaptosuccinic acid scan in acute pyelonephritis. Pediatr Nephrol, 1994. 8: 694696.

67. Nguyen, H.T., S.B. Bauer, C.A. Peters, L.P. Connolly, R. Gobet, J.G. Borer, C.E. Barnewolt, P.L. Ephraim, S.T. Treves, and A.B. Retik, 99m Technetium dimercapto-succinic acid renal scintigraphy abnormalities in infants with sterile high grade vesicoureteral reflux. J Urol, 2000. 164(5): 1674-8; discussion 1678-9.

68. Orsola De Los Santos, A., J. Caffaratti Sfulcini, and J.M. Garat Barredo, Valor de la gammagrafia renal con DMSA en las pielonefritis agudas en niños. Urol Integr Invest, 1997. 2: 192-198.

69. Parkhouse, H.F., M.L. Godley, J. Cooper, R.A. Risdon, and P.G. Ransley, Renal imaging with 99Tcm-labelled DMSA in the detection of acute pyelonephritis: an experimental study in the pig. Nucl Med Commun, 1989. 10(1): 63-70.

70. Majd, M. and H.G. Rushton, Renal cortical scintigraphy in the diagnosis of acute pyelonephritis. Semin Nucl Med, 1992. 22(2): 98-111.

71. Wallin, L. and M. Bajc, The significance of vesicoureteric reflux on kidney development assessed by dimercaptosuccinate renal scintigraphy. Br J Urol, 1994. 73(6): 607-11.

72. Melis, K., J. Vandevivere, C. Hoskens, A. Vervaet, A. Sand, and K.J. Van Acker, Involvement of the renal parenchyma in acute urinary tract infection: the contribution of $99 \mathrm{mTc}$ dimercaptosuccinic acid scan. Eur J Pediatr, 1992. 151(7): 536-9

73. Jakobsson, B., U. Berg, and L. Svensson, Renal scarring after acute pyelonephritis. Arch Dis Child, 1994. 70(2): 111-5. 
74. Goldarich, N.P. and I.H. Goldarich, Update on dimercaptosuccinic acid renal scaning in children with urinary tract infection. Pediatr Nephrol, 1995. 9: 21-226.

75. Rushton, H.G., M. Majd, B. Jantausch, B.L. Wiedermann, and A.B. Belman, Renal scarring following reflux and nonreflux pyelonephritis in children: evaluation with 99mtechnetium-dimercaptosuccinic acid scintigraphy. J Urol, 1992. 147(5): 1327-32.

76. Rushton, H.G., Jr., Vesicoureteral reflux-new concepts and techniques. J Urol, 1997. 157(4): 1414-5.

77. Jakobsson, B., S.H. Jacobson, and K. Hjalmas, Vesicoureteric reflux and other risk factors for renal damage: identification of high- and low-risk children. Acta Paediatr Suppl, 1999. 88(431): 31-9.

78. Slovis, T.L., Is there a single most appropriate imaging workup of a child with an acute febrile urinary tract infection? Pediatr Radiol, 1995. 25 Suppl 1: S46-9.

79. Hoberman, A., M. Charron, R.W. Hickey, M. Baskin, D.H. Kearney, and E.R. Wald, Imaging studies after a first febrile urinary tract infection in young children. N Engl J Med, 2003. 348(3): 195-202.

80. Elo, J., L.G. Tallgren, O. Alfthan, and S. Sarna, Character of urinary tract infections and pyelonephritic renal scarring after antireflux surgery. J Urol, 1983. 129(2):343-6.

81. Group, B.R.S., Prospective trial of operative versus nonoperative treatment of severe vesicoureteric reflux in children: five years' observation. Birmingham Reflux Study Group. Br Med J (Clin Res Ed), 1987. 295(6592): 237-41.

82. Olbing, H., I. Claesson, K.D. Ebel, U. Seppanen, J.M Smellie, T. Tamminen-Mobius, and I. Wikstad, Renal scars and parenchymal thinning in children with vesicoureteral reflux: a 5-year report of the International Reflux Study in Children (European branch). J Urol, 1992. 148(5 Pt 2): 1653-6.

83. Weiss, R., J. Duckett, and A. Spitzer, Results of a randomized clinical trial of medical versus surgical management of infants and children with grades III and IV primary vesicoureteral reflux (United States). The International Reflux Study in Children. J Urol, 1992. 148 (5 Pt 2): 1667-73.

84. Webster, R.I., G. Smith, R.H. Farnsworth, M.A. Rossleigh, A.R. Rosenberg, and G. Kainer, Low incidence of new renal scars after ureteral reimplantation for vesicoureteral reflux in children: a prospective study. J Urol, 2000. 163(6): 1915-8.

85. Piepsz, A., T. Tamminen-Mobius, C. Reiners, J. Heikkila, A. Kivisaari, N.J. Nilsson, R. Sixt, R.A. Risdon, J.M. Smellie, and B. Soderborg, Five-year study of medical or surgical treatment in children with severe vesico-ureteral reflux dimercaptosuccinic acid findings. International Reflux Study Group in Europe. Eur J Pediatr, 1998. 157(9): 753-8.

86. Aggarwal, V.K., K. Verrier Jones, A.W. Asscher, C. Evans, and L.A. Williams, Covert bacteriuria: long term follow up. Arch Dis Child, 1991. 66(11): 1284-6.

87. Bellinger, M.F. and J.W. Duckett, Vesicoureteral reflux: a comparison of non-surgical and surgical management. Contrib Nephrol, 1984. 39: 81-93.

88. Ben-Ami, T., L. Sinai, M. Hertz, and H. Boichis, Vesicoureteral reflux in boys: review of 196 cases. Radiology, 1989. 173(3): 681-4.

89. Burge, D.M., M.D. Griffiths, P.S. Malone, and J.D. Atwell, Fetal vesicoureteral reflux: outcome following conservative postnatal management. J Urol, 1992. 148(5 Pt 2): 1743-5.
90. Cardiff-Oxford Bacteriuria Study Group, C., Sequelae of covert bacteriuria in schoolgirls. A 4-year follow-up study. Lancet, 1978. 1: 889-93.

91. Edwards, D., I.C. Normand, N. Prescod, and J.M. Smellie, Disappearance of vesicoureteric reflux during long-term prophylaxis of urinary tract infection in children. Br Med J, 1977. 2(6082): 285-8.

92. Homsy, Y.L., I. Nsouli, B. Hamburger, I. Laberge, and E. Schick, Effects of oxybutynin on vesicoureteral reflux in children. J Urol, 1985. 134(6):. 1168-71.

93. Husmann, D.A. and T.D. Allen, Resolution of vesicoureteral reflux in completely duplicated systems: fact or fiction? J Urol, 1991. 145(5): 1022-3.

94. Jakobsen, B.E., H. Genster, S. Olesen, and E. Nygaard, Vesico-ureteral reflux in children. Br J Urol, 1977. 49(2): 119-27.

95. Koff, S.A. and D.S. Murtagh, The uninhibited bladder in children: effect of treatment on recurrence of urinary infection and on vesicoureteral reflux resolution. J Urol, 1983. 130(6): 1138-41.

96. Scholtmeijer, R.J., [Problems in the diagnosis and treatment of children with urologic disorders]. Ned Tijdschr Geneeskd, 1988. 132(19): 849-54.

97. Shah, K.J., D.G. Robins, and R.H. White, Renal scarring and vesicoureteric reflux. Arch Dis Child, 1978. 53(3): 210-7.

98. Smellie, J.M., U. Jodal, H. Lax, T.T. Mobius, H. Hirche, and $\mathrm{H}$. Olbing, Outcome at 10 years of severe vesicoureteric reflux managed medically: Report of the International Reflux Study in Children. J Pediatr, 2001. 139(5): 656-63.

99. Beetz, R., H. Schulte-Wissermann, J. Troger, H. Riedmiller, W. Mannhardt, O. Schofer, and R. Hohenfellner, Long-term follow-up of children with surgically treated vesicorenal reflux: postoperative incidence of urinary tract infections, renal scars and arterial hypertension. Eur Urol, 1989. 16(5): 366-71.

100. Carpentier, P.J., P.J. Bettink, W.C. Hop, and F.H. Schroder, Reflux-a retrospective study of 100 ureteric reimplantations by the Politano-Leadbetter method and 100 by the Cohen technique. Br J Urol, 1982. 54(3): 230-3.

101. Hjalmas, K., G. Lohr, T. Tamminen-Mobius, J. Seppanen, H. Olbing, and S. Wikstrom, Surgical results in the International Reflux Study in Children (Europe). J Urol, 1992. 148(5 Pt 2): 1657-61.

102. Scholtmeijer, R.J. and D.J. Griffiths, Treatment of vesicoureteric reflux. Preliminary report of a prospective study. Br J Urol, 1988. 61(3): 205-9.

103. Scott, D.J., H.N. Blackford, M.R. Joyce, A.R. Mundy, C.H. Kinder, G.B. Haycock, and C. Chantler, Renal function following surgical correction of vesico-ureteric reflux in childhood. Br J Urol, 1986. 58(2): 119-24.

104. Schiepers, C., L. Mesotten, W. Proesmans, R. Vereecken, A. Verbruggen, and M. de Roo, Surgical correction of vesicoureteral reflux: 5-year follow-up with 99Tcm-DMSA scintigraphy. Nucl Med Commun, 2001. 22(2): 217-24.

105. Steffens, J., P.H. Langen, B. Haben, R. Hiebl, L. Steffens, and M.S. Polsky, Politano-leadbetter ureteroneocystostomy. A 30-year experience. Urol Int, 2000. 65(1): 9-14.

106. Choi, H., S.J. Oh, Y. So, D.S. Lee, A. Lee, and K.M. Kim, No further development of renal scarring after antireflux surgery in children with primary vesicoureteral reflux: review of the results of 99mtechnetium dimercapto-succinic acid renal scan. J Urol, 1999. 162(3 Pt 2): 1189-92. 
107. Lackgren, G., N. Wahlin, and A. Stenberg, Endoscopic treatment of children with vesico-ureteric reflux. Acta Paediatr Suppl, 1999. 88(431): 62-71.

108. Lyon, R.P., Renal arrest. J Urol, 1973. 109(4): 707-10.

109. Redman, J.F., L.J. Scriber, and N.K. Bissada, Apparent failure of renal growth secondary to vesicoureteral reflux. Urology, 1974. 3(6): 704-7.

110. McRae, C.U., F.T. Shannon, and W.L. Utley, Effect on renal growth of reimplantation of refluxing ureters. Lancet, 1974. 1(7870): 1310-2.

111. Pinter, A.B., V. Jaszai, and I. Dober, Medical treatment of vesicoureteral reflux detected in infancy. J Urol, 1988. 140(1): 121-4.

112. Herndon, C.D., P.H. McKenna, T.F. Kolon, E.T. Gonzales, L.A. Baker, and S.G. Docimo, A multicenter outcomes analysis of patients with neonatal reflux presenting with prenatal hydronephrosis. J Urol, 1999. 162(3 Pt 2): 1203-8.

113. Olbing, H., H. Hirche, O. Koskimies, H. Lax, U. Seppanen, J.M. Smellie, T. Tamminen-Mobius, and I. Wikstad, Renal growth in children with severe vesicoureteral reflux: 10-year prospective study of medical and surgical treatment: the International Reflux Study in Children (European branch). Radiology, 2000. 216(3): 731-7.

114. Berg, U.B., Long-term followup of renal morphology and function in children with recurrent pyelonephritis. J Urol, 1992. 148(5 Pt 2): 1715-20.

115. Smellie, J.M., T.M. Barratt, C. Chantler, I. Gordon, N.P. Prescod, P.G. Ransley, and A.S. Woolf, Medical versus surgical treatment in children with severe bilateral vesicoureteric reflux and bilateral nephropathy: a randomised trial. Lancet, 2001. 357(9265): 1329-33.

116. Poulsen, E.U., N.L. Johannesen, J.B. Nielsen, T.M. Jorgensen, and A.J. Andersen, Vesico-ureteral reflux. II. The longterm outcome of kidney function in non-surgical treatment. Scand J Urol Nephrol Suppl, 1989. 125: 2934.

117. Pohl, H.G., H.G. Rushton, J.S. Park, R. Chandra, and M. Majd, Adjunctive oral corticosteroids reduce renal scarring: the piglet model of reflux and acute experimental pyelonephritis. J Urol, 1999. 162(3 Pt 1): 815-20.

118. Beetz, R., W. Mannhardt, M. Fisch, R. Stein, and J.W. Thuroff, Long-term followup of 158 young adults surgically treated for vesicoureteral reflux in childhood: the ongoing risk of urinary tract infections. J Urol, 2002. 168(2): 704-7; discussion 707.

119. Hanson, E., S. Hansson, and U. Jodal, Trimethoprimsulphadiazine prophylaxis in children with vesicoureteric reflux. Scand J Infect Dis, 1989. 21(2): 201-4.

120. Mansfield, J.T., B.W. Snow, P.C. Cartwright, and K. Wadsworth, Complications of pregnancy in women after childhood reimplantation for vesicoureteral reflux: an update with 25 years of followup. J Urol, 1995. 154(2 Pt 2): 787-90.

121. Lewis, M.A., Report of the Paediatric Renal Registry. In: Answell D, Feest T. The Second Annual Report of the UK Renal registry. Bristol Renal Association, 1999:157-8.

122. Wallace, D.M., D.L. Rothwell, and D.I. Williams, The longterm follow-up of surgically treated vesicoureteric reflux. Br J Urol, 1978. 50(7): 479-84.

123. Lenaghan, D., J.G. Whitaker, F. Jensen, and D. Stephens, The natural histiry of reflux and long-term effects of reflux on the kidney. J Urol, 1976. 115: 728-30.
124. Nielsen, J.B., J. Frokiaer, M. Rehling, T.M. Jorgensen, and J.C. Djurhuus, A 14-year follow-up of conservative treatment for vesico-ureteric reflux. BJU Int, 2000. 86(4): 502-7.

125. Kohler, J.R., J. Tencer, H. Thysell, L. Forsberg, and M. Hellstrom, Long-term effects of reflux nephropathy on blood pressure and renal function in adults. Nephron Clin Pract, 2003. 93(1): C35-46.

126. Goonasekera, C.D. and M.J. Dillon, Hypertension in reflux nephropathy. BJU Int, 1999. 83 Suppl 3: 1-12.

127. Zucchelli, P. and R. Gaggi, Vesicoureteral reflux and reflux nephropathy in adults. Contrib Nephrol, 1988. 61: 210-9.

128. Kincaid-Smith, P. and G. Becker, Reflux nephropathy and chronic atrophic pyelonephritis: a review. J Infect Dis, 1978. 138(6): 774-80.

129. Dwoskin, J.Y. and A.D. Perlmutter, Vesicoureteral reflux in children: a computerized review. J Urol, 1973. 109(5): 888-90.

130. Polito, C., A. La Manna, A. Capacchione, F. Pullano, A. Iovene, and R. Del Gado, Height and weight in children with vesicoureteric reflux and renal scarring. Pediatr Nephrol, 1996. 10(5): 564-7.

131. Smellie, J.M., M.A. Preece, and A.M. Paton, Normal somatic growth in children receiving low-dose prophylactic co-trimoxazole. Eur J Pediatr, 1983. 140(4):. 301-4.

132. Merrell, R.W. and J.J. Mowad, Increased physical growth after successful antireflux operation. J Urol, 1979. 122(4): 523-7.

133. Polito, C., A. Marte, M. Zamparelli, M.R. Papale, C.E. Rocco, and A. La Manna, Catch-up growth in children with vesicoureteric reflux. Pediatr Nephrol, 1997. 11(2): 164-8.

134. Astley, R., R.C. Clrack, J.J. Corkery, and e. al., Prospective trial of operative versus nonoperative treatment of severe vesicoureteric reflux in children:two years ${ }^{-}$observation in 96 children. BMJ, 1983. 287: 171-174.

135. Astley, R., R.C. Clark, J.J. Corkery, and e. al., Prospective trial of operative versus nonoperative treatment of severe vesicoureteric reflux in children:five years ${ }^{-}$observation in 96 children. BMJ, 1987. 295: 237-241.

136. Golan, A., S. Wexler, A. Amit, D. Gordon, and M.P. David, Asymptomatic bacteriruia in normal and high-risk pregnancy. Eur J Obst. Gynec. Reprod. Biol., 1989. 33: 101.

137. Sweet, R.L., Bacteriruia and pyelonephritis during pregnancy. Semin Perinatol, 1977. 1: 25-40.

138. Kass, E.H., Bacteriruia and pyelonephritis of pregnancy. Arch . Inte. Med., 1960. 105: 194.

139. Martens, M.G., Pyeloneprhitis. Obst. Gynec. Clin. N. Amer., 1989. 16: 305.

140. Martinell, J., I. Claesson, G. Lidin-Janson, and U. Jodal, Urinary infection, reflux and renal scarring in females continuously followed for 13-38 years. Pediatr Nephrol, 1995. 9(2): 131-6.

141. McGladdery, S.L., S. Aparicio, K. Verrier-Jones, R. Roberts, and S.H. Sacks, Outcome of pregnancy in an Oxford-Cardiff cohort of women with previous bacteriuria. Q J Med, 1992. 83(303):. 533-9.

142. Martinell, J., G. Lidin-Janson, R. Jagenburg, R. Sivertsson, I. Claesson, and U. Jodal, Girls prone to urinary infections followed into adulthood. Indices of renal disease. Pediatr Nephrol, 1996. 10(2):139-42.

143. Romero, R., E. Oyarzun, M. Mazor, and M. Sirtori, Metaanalysis of relationship between asymptomatic bacteriuria and preterm delivery/low birth weight. Obstet Gynecol, 1989. 73:576-82. 
144. Kincaid-Smith, P. and K.F. Fairley, Renal disease in pregnancy. Three controversial areas: mesangial IgA nephropathy, focal glomerular sclerosis (focal and segmental hyalinosis and sclerosis), and reflux nephropathy. Am J Kidney Dis, 1987. 9(4):328-33.

145. Cunningham, F.G., S.M. Cox, T.W. Harstad, R.A. Masson, and J.A. Pitchard, Chronic renal disease and pregnancy outcome. Am J Obstet Gynecol, 1990. 163:453-9.

146. Williams, G., D. Davies, K.T. Evans, and J.E. Williams, Vesicoureteric reflux in patients with bacteriruia in pregnancy. Lancet, 1968. 4:1202-5.

147. Bukowski, T.P., G.G. Betrus, J.W. Aquilina, and A.D. Perlmutter, Urinary tract infections and pregnancy in women who underwent antireflux surgery in childhood. J Urol, 1998. 159(4):1286-9.

148. Fryczkowski, M., Results of operative treatment of vesicoureteral reflux by the Leadbetter-Politano method in children. Int Urol Nephrol, 1976. 8(1):41-6.

149. Austenfeld, M.S. and B.W. Snow, Complications of pregnancy in women after reimplantation for vesicoureteral reflux. J Urol, 1988. 140(5 Pt 2):1103-6.

150. Orikasa, S., A new antireflux operation. Eur Urol, 1990. 17(4):330-2.

151. Ehrlich, R.M., The ureteral folding technique for megaureter surgery. J Urol, 1985. 134(4):668-70.

152. Hagberg, S., K. Hjalmas, B. Jacobsson, and U. Sillen, Renal growth after antireflux surgery in infants. $Z$ Kinderchir, 1984. 39(1):52-4.

153. Maggiolo, L.F., J.L. Lockhart, and V.A. Politano, Management of massively refluxing megaureters. Urology, 1983. 21(3):232-5.

154. Ahmed, S. and H. Tan, Complications of transverse advancement ureteral reimplantation: diverticulum formation. J Urol, 1982. 127(5):970-3.

155. Arap, S., E.G. Abrao, and G. Menezes de Goes, Treatment and prevention of complications after extravesical antireflux technique. Eur Urol, 1981. 7(5): 263-7.

156. Broaddus, S.B., P.M. Zickerman, P.M. Morrisseau, and G.W. Leadbetter, Jr., Incidence of later ureteral obstruction after antireflux surgery in infants and children. Urology, 1978. 11(2): 139-41.

157. Hampel, N., D. Richter-Levin, and I. Gersh, Extravesical repair of primary vesicoureteral reflux in children. J Urol, 1977. 117(3): 355-7.

158. Willscher, M.K., S.B. Bauer, P.J. Zammuto, and A.B. Retik, Renal growth and urinary infection following antireflux surgery in infants and children. J Urol, 1976. 115(6): 722-5

159. Govan, D.E., W.R. Fair, G.W. Friedland, and R.A. Filly, Management of children with urinary tract infections: the Stanford experience. Urology, 1975. 6(3): 273-86.

160. Jonas, P., M. Many, H. Boichis, and M. Hertz, Ureteroneocystostomy in children with vesicoureteral reflux. Experience with 150 reimplanted ureters. Isr J Med Sci, 1974. 10(6): 603-7.

161. Garrett, R.A. and D.P. Schlueter, Complications of antireflux operations: causes and management. J Urol, 1973. 109(6): 1002-4.

162. Duckett, J.W., R.D. Walker, and R. Weiss, Surgical results: International Reflux Study in Children-United States branch. J Urol, 1992. 148(5 Pt 2): 1674-5.

163. Wacksman, J., A. Gilbert, and C.A. Sheldon, Results of the renewed extravesical reimplant for surgical correction of vesicoureteral reflux. J Urol, 1992. 148(2 Pt 1): 359-61.
164. Burbige, K.A., Ureteral reimplantation: a comparison of results with the cross-trigonal and Politano-Leadbetter techniques in 120 patients. J Urol, 1991. 146(5): 1352-3.

165. Bradic, I., S. Batinica, and J. Husar, Primary vesicoureteric reflux treated by antireflux ureterocystostomy at the vertex of the bladder. A 12-year follow-up and analysis of operative failure. $\mathrm{Br} \mathrm{J}$ Urol, 1988. 62(6): 531-6.

166. Sutton, R. and J.D. Atwell, Physical growth velocity during conservative treatment and following subsequent surgical treatment for primary vesicoureteric reflux. $\mathrm{Br} J$ Urol, 1989. 63(3): 245-50.

167. Pypno, W., Antireflux operations without catheter. Int Urol Nephrol, 1987. 19(2): 141-3.

168. Zaontz, M.R., M. Maizels, E.C. Sugar, and C.F. Firlit, Detrusorrhaphy: extravesical ureteral advancement to correct vesicoureteral reflux in children. J Urol, 1987. 138(4 Pt 2): 947-9.

169. Kondo, A. and T. Otani, Correction of reflux with the ureteric crossover method. Clinical experience in 50 patients. Br J Urol, 1987. 60(1): 36-8.

170. Ehrlich, R.M., Vesicoureteral reflux: a surgeon's perspective. Pediatr Clin North Am, 1982. 29(4): 827-34.

171. Faure, G., S. Ben Salah, P.L. d'Escoffier, and M. Revol, Bilateral Cohen's antireflux procedure with a single submucosal tunnel. Eur Urol, 1982. 8(4): 193-5.

172. Mundy, A.R., C.H. Kinder, M.R. Joyce, C. Chantler, and G.B. Haycock, Improvement in renal function following ureteric reimplantation for vesicoureteric reflux. $\mathrm{Br} \mathrm{J}$ Urol, 1981. 53(6): 542-4.

173. Hanna, M.K., Early surgical correction of massive refluxing megaureter in babies by total ureteral reconstruction and reimplantation. Urology, 1981. 18(6): 562-6.

174. McDuffie, R.W., R.B. Litin, and K.E. Blundon, Ureteral reimplantation: Lich method. Urology, 1977. 10(1): 1922.

175. Johnston, J.H. and A. Farkas, The congenital refluxing megaureter: experiences with surgical reconstruction. $\mathrm{Br}$ J Urol, 1975. 47(2): 153-9.

176. Ravasini, G. and F. Pagano, Surgical correction of vesicoureteral reflux. Description of technique and results. Urol Int, 1973. 28(1): 56-64.

177. Allen, T.D., Modification of the ureteral advancement procedure for vesicoureteral reflux. South Med J, 1973. 66(3): 305-7.

178. Houle, A.M., G.A. McLorie, D.M. Heritz, P.H. McKenna, B.M. Churchill, and A.E. Khoury, Extravesical nondismembered ureteroplasty with detrusorrhaphy: a renewed technique to correct vesicoureteral reflux in children. J Urol, 1992. 148(2 Pt 2): 704-7.

179. Dietz, H.G., A. Schmidt, J.B. Bader, and A. Markus, The Politano-Leadbetter antireflux plasty. Investigation of complications in 245 children. Eur J Pediatr Surg, 1996. 6(5): 277-80.

180. Burbige, K.A., M. Miller, and J.P. Connor, Extravesical ureteral reimplantation: results in 128 patients. J Urol, 1996. 155(5): 1721-2.

181. Dodat, H., A.F. Valmalle, J.D. Weidmann, F. Collet, G. Pelizzo, and R. Dubois, [Endoscopic treatment of vesicorenal reflux in children. Five-year assessment of the use of Macroplastique]. Prog Urol, 1998. 8(6): 1001-6.

182. King, P.A. and I. Gollow, The endoscopic correction of vesico-ureteric reflux. Aust N Z J Surg, 1988. 58(7): 56971. 
183. Sweeney, L.E. and P.S. Thomas, Evaluation of subureteric Teflon injection as an antireflux procedure. Ann Radiol (Paris), 1987. 30(7): 478-81.

184. Dewan, P.A., Re: Evaluation of polydimethylsiloxane as an alternative in the endoscopic treatment of vesicoureteral reflux. J Urol, 1995. 153(5): 1644-5.

185. Kaminetsky, J.C. and M.K. Hanna, Endoscopic treatment of vesicoureteral reflux in children with neurogenic bladders. Urology, 1991. 37(3): 244-7.

186. Frey, P., N. Lutz, P. Jenny, and B. Herzog, Endoscopic subureteral collagen injection for the treatment of vesicoureteral reflux in infants and children. J Urol, 1995 154(2 Pt 2): 804-7.

187. Gómez Fraile, A., A. Aransay Brantot, y F. López Vázquez, Tratamiento endoscópico del reflujo vésico-ureteral en niños menores de un año. Urol Integr Invest, 2002. 7(4):. 494-500.

188. El-Khatib, M., D.K. Packham, G.J. Becker, and P. Kincaid-Smith, Pregnancy-related complications in women with reflux nephropathy. Clin Nephrol, 1994. 41(1): 50-5.

189. Puri, P., G.K. Ninan, and R. Surana, Subureteric Teflon injection (STING). Results of a European survey. Eur Urol, 1995. 27(1): 71-5.

190. Stenberg, A. and G. Lackgren, A new bioimplant for the endoscopic treatment of vesicoureteral reflux: experimental and short-term clinical results. J Urol, 1995. 154(2 Pt 2): $800-3$.

191. Vuckov, S., H. Nikolic, A. Kvesic, and N. Bukvic, Our experience in the treatment of the vesico-ureteral reflux with Lich-Gregoir antireflux surgical procedure. Eur J Pediatr Surg, 1999. 9(1): 33-6.

192. Leissner, J., E.P. Allhoff, W. Wolff, C. Feja, M. Hockel, P. Black, and R. Hohenfellner, The pelvic plexus and antireflux surgery: topographical findings and clinical consequences. J Urol, 2001. 165(5): 1652-5.

193. Lipski, B.A., M.E. Mitchell, and M.W. Burns, Voiding dysfunction after bilateral extravesical ureteral reimplantation. J Urol, 1998. 159(3): 1019-21.

194. Minevich, E., D. Aronoff, J. Wacksman, and C.A. Sheldon, Voiding dysfunction after bilateral extravesical detrusorrhaphy. J Urol, 1998. 160(3 Pt 2): 1004-6; discussion 1038

195. Ross, J.H., R. Kay, and P. Nasrallah, Contralateral reflux after unilateral ureteral reimplantation in patients with a history of resolved contralateral reflux. J Urol, 1995. 154(3): 1171-2.

196. Kumar, R. and P. Puri, Newly diagnosed contralateral reflux after successful unilateral endoscopic correction: is it due to the pop-off mechanism? $\mathrm{J}$ Urol, 1997. 158(3 Pt 2): 1213-5.

197. Minevich, E., J. Wacksman, A.G. Lewis, and C.A. Sheldon, Incidence of contralateral vesicoureteral reflux following unilateral extravesical detrusorrhaphy (ureteroneocystostomy). J Urol, 1998. 159(6): 2126-8.

198. Sargin, S.Y., A. Ergen, H.A. Ozen, H. Ozkardes, S. Tekgul, I. Erkan, and M. Bakkaloglu, The fate of contralateral ureter after ipsilateral reimplantation in unilateral vesicoureteric reflux. Int Urol Nephrol, 1991. 23(1):. 31-5.

199. Cain, M.P., D.A. Husmann, R.H. McLaren, and S.A. Kramer, Continuous epidural anesthesia after ureteroneocystostomy in children. J Urol, 1995. 154(2 Pt 2): 791-3.
200. Park, J.M., C.S. Houck, N.F. Sethna, L.J. Sullivan, A. Atala, J.G. Borer, B.G. Cilento, D.A. Diamond, C.A. Peters, A.B. Retik, and S.B. Bauer, Ketorolac suppresses postoperative bladder spasms after pediatric ureteral reimplantation. Anesth Analg, 2000. 91(1): 11-5.

201. El-Ghoneimi, A., C. Deffarges, R. Hankard, F. JeanEudes, Y. Aigrain, and E. Jacqz-Aigrain, Intravesical morphine analgesia is not effective after bladder surgery in children: results of a randomied double-blind study. $J$ Urol, 2002. 168(2): 694-7.

202. So, E.P., W.A. Brock, and G.W. Kaplan, Ureteral reimplantation without catheters. J Urol, 1981. 125(4): 551-3.

203. Fort, K.F., S.H. Selman, and K.A. Kropp, A retrospective analysis of the use of ureteral stents in children undergoing ureteroneocystotomy. J Urol, 1983. 129(3): 545-7.

204. Remzi, D., H.A. Ozen, I. Erkan, and S. Kendi, 15 years of experience in the surgical treatment of vesicoureteral reflux in children. Turk J Pediatr, 1984. 26(1-4): 175-9.

205. Brandell, R.A. and J.W. Brock, 3rd, Ureteral reimplantation: postoperative management without catheters. Urology, 1993. 42(6): 705-7.

206. Puri, P., Endoscopic correction of vesicoureteral reflux. Curr Opin Urol, 2000. 10(6): 593-7.

207. Granata, C., P. Buffa, E. Di Rovasenda, G. Mattioli, P.L. Scarsi, E. Podesta, P. Dodero, and V. Jasonni, Treatment of vesico-ureteric reflux in children with neuropathic bladder: a comparison of surgical and endoscopic correction. J Pediatr Surg, 1999. 34(12):1836-8.

208. Dirección General de Salud Pública, D., Guía de las indicaciones para la correcta solicitud de pruebas de diagnóstico por imagen., ed. C. Europeas. 2001, Madrid Ministerio de Sanidad y Consumo. 19-33.

209. Zerin, J.M. and B. Shulkin, Postprocedural symptoms in children who undergo imaging studies of the urinary tract: is it the constrast material or the catheter? Radiology, 1992. 182: 727-30.

210. Elder, J.S., Guidelines for consideration for surgical repair of vesicoureteral reflux. Curr Opin Urol, 2000. 10(6): 579-85.

211. Gooding, C., W.E. Berdon, A. Brodeur, and M. Rowen, Adverse reactions to intravenous pyelography in children. AJR Am J Roentgenol, 1975. 123: 802-4.

212. Bisset, G.S., 3rd, J.L. Strife, and J.S. Dunbar, Urography and voiding cystourethrography: findings in girls with urinary tract infection. AJR Am J Roentgenol, 1987. 148(3): 479-82.

213. Abrams, P.H., R.B. Freeman, C. Anderström, and A. Matiasson, Tolterodine a new antimuscarinic agent:as effective but better tolerated than oxybutynin in patients with overactive bladder. Br J Urol, 1998. 81(6): 801-810.

214. Bolduc, S., J. Upadhyay, J. Payton, D. Bagli, G. McLorie, A. Khoury, and W. Farhat, The use of tolterodine in children after oxybutynin faliure. BJU Int, 2003. 91(4): 398401

215. Savage, D.C., M.I. Wilson, and E. Ross, Asymptomatic bacteriuria in girls entrants to Dundee primary schools. BMJ, 1969. 3: 75-80.

216. McLachlan, M.S., S.T. Meller, E.R. Jones, A.W. Asscher, E.W. Fletcher, R.T. Mayon-White, J.G. Ledingham, J.C. Smith, and H.H. Johnston, Urinary tract in schoolgirls with covert bacteriuria. Arch Dis Child, 1975. 50(4): 253-8. 
217. Claesson, I. and U. Lindberg, Asymptomatic bacteriuria in schoolgirls. VII. A follow-up study of the urinary tract in treated and untreated schoolgirls with asymptomatic bacteriuria. Radiology, 1977. 124(1): 179-83.

218. Ditchfield, M.R., J.F. De Campo, D.J. Cook, T.M. Nolan, H.R. Powell, R. Sloane, K. Grimwood, and S. Cahill, Vesicoureteral reflux: an accurate predictor of acute pyelonephritis in childhood urinary tract infection? Radiology, 1994. 190(2):.413-5.

219. Rosenberg, A.R., M.A. Rossleigh, M.P. Brydon, S.J. Bass, D.M. Leighton, and R.H. Farnsworth, Evaluation of acute urinary tract infection in children by dimercaptosuccinic acid scintigraphy: a prospective study. J Urol, 1992. 148(5 Pt 2): 1746-9.

220. Sargent, M.A. and D.A. Stringer, Voiding cystourethrography in children with urinary tract infection: the frequency of vesicoureteric reflux is independent of the specialty of the physician requesting the study. AJR Am J Roentgenol, 1995. 164(5): 1237-41.

221. Masson, W., Urinary tract infectins in children:renal ultrasound evaluation. Radiology, 1984. 153:109-111.

222. Strife, J.L., G.S. Bisset, 3rd, D.R. Kirks, F.J. Schlueter, M.J. Gelfand, D.S. Babcock, and B.K. Han, Nuclear cystography and renal sonography: findings in girls with urinary tract infection. AJR Am J Roentgenol, 1989. 153(1): 115-9.

223. Blickman, J.G., C.M. Taylor, and R. Lebowitz, Voiding cystourethrography:the initial radiologic study in children with urinary tract infection. Radiology, 1985. 156:.659-662.

224. McKerrow, W., N. Davidson-Lamb, and P.F. Jones, Urinary tract infection in children. Br Med J (Clin Res Ed), 1984. 289(6440): 299-303.

225. DiPietro, M.A., C.E. Blane, and J.M. Zerin, Vesicoureteral reflux in older children: concordance of US and voiding cystourethrographic findings. Radiology, 1997. 205(3):821-2.

226. Wennerstrom, M., S. Hansson, U. Jodal, and E. Stokland, Disappearance of vesicoureteral reflux in children. Arch Pediatr Adolesc Med, 1998. 152(9):879-83.

227. Hellstrom, M., B. Jacobsson, S. Marild, and U. Jodal, Voiding cystourethrography as a predictor of reflux nephropathy in children with urinary-tract infection. AJR Am J Roentgenol, 1989. 152(4):801-4.

228. Craig, J.C. and L.M. Irwig, Symptomatic urinary tract infection in prescool Australian children. J Paediatr Child Health, 1998. 34: 154-159.

229. Lavocat, M.P., D. Granjon, D. Allard, C. Gay, M.T Freycon, and F. Dubois, Imaging of pyelonephritis. Pediatr Radiol, 1997. 27(2): 159-65.

230. Stockland, E., A.L. Hellstrom, and B. Jacobson, Renal damage one year after first urinary trast infection: role of dimercaptosuccinic acid scintigraphy. J Pediatr, 1996. 129:815-820.

231. Benador, D., N. Benador, D. Slosman, B. Mermillod, and E. Girardin, Are younger children at highest risk of renal sequelae after pyelonephritis? Lancet, 1997. 349(9044): 17-9.

232. Bjorgvinsson, E., M. Majd, and D.F. Eggli, Diagnosis of acute pyelonephritis in children:comparison of sonography and 99m technetium-DMSA scintigraphy. AJR Am J Roentgenol, 1991. 157:539-543.

233. Kass, E.J., D. Fink-Bennett, and A. Cacciarelli, The sensitivity of renal scintagraphy and sonography in detecting nonobstructive acute pyelonephritis. J Urol, 1992. 148:606-608.
234. Greenfield, S.P. and E. Afshani, Vesicoureteral reflux in children with and without a history of urinary tract infection: a comparative analysis. Urology, 1992. 40(4): 33942.

235. Polito, C., P.F. Rambaldi, A. La Manna, L. Mansi, and R. Di Toro, Enhanced detection of vesicoureteric reflux with isotopic cystography. Pediatr Nephrol, 2000. 14(8-9):82730 .

236. Elder, J.S., H.M. Snyder, C. Peters, B. Arant, C.E. Hawtrey, R.S. Hurwitz, T.S. Parrott, and R.A. Weiss, Variations in practice among urologists and nephrologists treating children with vesicoureteral reflux. J Urol, 1992. 148(2 Pt 2):714-7.

237. Elder, J.S., Antenatal hydronephrosis. Fetal and neonatal management. Pediatr Clin North Am, 1997. 44(5): 1299-321.

238. Hollowell, J.G. and S.P. Greenfield, Screening siblings for vesicoureteral reflux. J Urol, 2002. 168(5): 2138-41.

239. Puri, P., Controversies in the management of vesicoureteric reflux. Ir Med J, 1992. 85(4 Suppl): 4-6.

240. Sargent, M.A., What is the normal prevalence of vesicoureteral reflux? Pediatr Radiol, 2000. 30(9):587-93.

241. Peppas, D.S., S.J. Skoog, D.A. Canning, and A.B. Belman, Nonsurgical management of primary vesicoureteral reflux in complete ureteral duplication: is it justified? J Urol, 1991. 146(6): 1594-5.

242. Zerin, J.M. and J. Leiser, The impact of vesicoureteral reflux on contralateral renal length in infants with multicystic dysplastic kidney. Pediatr Radiol, 1998. 28(9): 83-6.

243. Olbing, $H$. and $T$. Tamminen-Mobius, International reflux study in children. Contrib Nephrol, 1988. 61: 228-35.

244. Atala, A., L.G. Cima, W. Kim, K.T. Paige, J.P. Vacanti, A.B. Retik, and C.A. Vacanti, Injectable alginate seeded with chondrocytes as a potential treatment for vesicoureteral reflux. J Urol, 1993. 150(2 Pt 2): 745-7.

245. Atala, A., W. Kim, K.T. Paige, C.A. Vacanti, and A.B. Retik, Endoscopic treatment of vesicoureteral reflux with a chondrocyte-alginate suspension. J Urol, 1994. 152(2 Pt 2): 641-3; discussion 644.

246. Diamond, D.A. and A.A. Caldamone, Endoscopic correction of vesicoureteral reflux in children using autologous chondrocytes: preliminary results. J Urol, 1999. 162(3 Pt 2): 1185-8.

247. Senuma, Y., S. Franceschin, J.G. Hilborn, P. Tissieres, I. Bisson, and P. Frey, Bioresorbable microspheres by spinning disk atomization as injectable cell carrier: from preparation to in vitro evaluation. Biomaterials, 2000. 21(11):1135-44.

248. Joyner, B.D. and A. Atala, Endoscopic substances for the treatment of vesicoureteral reflux. Urology, 1997. 50(4): 489-94.

249. El Fassi, M.J., T. Karmouni, K. Tazi, K. Khader, A. Koutani, A.A. Iben Attya, M. Hachimi, and A. Lakrissa, [Endoscopic treatment of vesico-ureteral reflux by submeatic injection of autologous fat. Two case reports]. Ann Urol (Paris), 2002. 36(2): 115-9.

250. Kershen, R.T. and A. Atala, New advances in injectable therapies for the treatment of incontinence and vesicoureteral reflux. Urol Clin North Am, 1999. 26(1): 81-94, VIII.

251. Palma, P.C., U. Ferreira, O. Ikari, and N. Rodrigues Netto, $\mathrm{Jr}$., Subureteric lipoinjection for vesicoureteral reflux in renal transplant candidates. Urology, 1994. 43(2): 174-7. 
252. Cedron, M., D.P. DeVore, and R. Connolly, The biological behavior of autologous collagen injected into the rabbit bladder. J Urol, 1995. 154:. 808.

253. O'Donnell, B. and P. Puri, Treatment of vesicoureteric reflux by endoscopic injection of Teflon. 1984. J Urol, 2002. 167(4): 1808-9; discussion 1810.

254. Aubert, D., O. Destuynder, and G. Zoupanos, [Treatment of vesico-renal reflux in children with endoscopic injection of polytetrafluoroethylene (teflon). Apropos of a series of 100 cases of reflux in 72 children]. Ann Urol (Paris), 1989. 23(5): 367-72.

255. Blake, N.S. and E. O'Connell, Endoscopic correction of vesico-ureteric reflux by subureteric Teflon injection: follow-up ultrasound and voiding cystography. $\mathrm{Br} \mathrm{J}$ Radiol, 1989. 62(737): 443-6.

256. Capozza, N., M. Patricolo, A. Lais, E. Matarazzo, and P. Caione, Endoscopic treatment of vesico-ureteral reflux: twelve years' experience. Urol Int, 2001. 67(3): 228-31.

257. Dessouki, T., F. Staerman, A. Abbar, M. Dawhara, and A. Gelet, Treatment of primary vesicoureteric reflux by polytetrafluoroethylene injection: a middle-term follow-up study. Eur Urol, 1993. 23(3): 375-8.

258. Lopez Lopez, J., J. Sanchez Zalabardo, M. Sanchez Elipe, J. Valdivia Uria, J. Valle Gerhold, and A. Jimenez Vidal, [Endoscopic treatment of vesicoureteral reflux]. An Esp Pediatr, 2001. 54(2):132-5.

259. Chaffange, P., R. Dubois, A. Bouhafs, A.F. Valmalle, and H. Dodat, [Endoscopic treatment of vesicorenal reflux in children: short- and long-term results of polytetrafluoroethylene (Teflon) injections]. Prog Urol, 2001. 11(3): 546-51.

260. Puri, P., Ten year experience with subureteric Teflon (polytetrafluoroethylene) injection (STING) in the treatment of vesico-ureteric reflux. Br J Urol, 1995. 75(2):12631.

261. Puri, P., M. Palanimuthu, and L. Dass, Endoscopic treatment of primary vesicoureteric reflux in infants by subureteric injection of polytetrafluoroethylene. A 9-year follow-up. Eur Urol, 1995. 27(1):67-70.

262. Mittleman, R.E., J. Marraccini, and A. Khoury, Pulmonary teflon granulomas following periurethral Teflon for urinary incontinence. Arch Pathol Lab Med, 1990. 107:611.

263. Borgatti, R., A. Tettamanti, and P. Piccinelli, Brain injury in a healthy child one year after periureteral injection of Teflon. Pediatrics, 1996. 98(2 Pt 1): 290-1.

264. Aaronson, I.A., R.A. Rames, W.B. Greene, L.G. Walsh, U.A. Hasal, and P.D. Garen, Endoscopic treatment of reflux: migration of Teflon to the lungs and brain. Eur Urol, 1993. 23(3):. 394-9.

265. Frey, P., D. Berger, P. Jenny, and B. Herzog, Subureteral collagen injection for the endoscopic treatment of vesicoureteral reflux in children. Followup study of 97 treated ureters and histological analysis of collagen implants. J Urol, 1992. 148(2 Pt 2):718-23.
266. Leonard, M.P., D.A. Canning, C.A. Peters, J.P. Gearhart, and R.D. Jeffs, Endoscopic injection of glutaraldehyde cross-linked bovine dermal collagen for correction of vesicoureteral reflux. J Urol, 1991. 145(1):115-9.

267. Leonard, M.P., A. Decter, L.W. Mix, H.W. Johnson, and G.U. Coleman, Endoscopic treatment of vesicoureteral reflux with collagen: preliminary report and cost analysis. J Urol, 1996. 155(5): 1716-20.

268. Frey, P., N. Lutz, D. Berger, and B. Herzog, Histological behavior of glutaraldehyde cross-linked bovine collagen injected into the human bladder for the treatment of vesicoureteral reflux. J Urol, 1994. 152(2 Pt 2):632-5.

269. Cukier, J., R. Beauchamp, and J. Spindler, Association between bovine collagen dermal implants and a dermatomyositis or a polymyositis-like sindrome. Ann Intern Med, 1993. 118: 920.

270. Cukier, J., [Vesico-renal reflux in adults]. J Urol Nephrol (Paris), 1977. 83 Suppl 2: 477-8.

271. Frankenschmidt, A., A. Katzenwadel, and L.B. Zimmerhack, Endoscopic treatment of relux by subureteric collagen injection:critical review of 5 years'experience. J Endourol, 1997. 11: 343.

272. Shah, N., M.J. Kabir, T. Lane, S. Avenell, and P.J. Shah, Vesico-ureteric reflux in adults with neuropathic bladders treated with Polydimethylsiloxane (Macroplastique). Spinal Cord, 2001. 39(2): 92-6.

273. Smith, D.P., W.E. Kaplan, and R. Oyasu, Evaluation of polydimethylsiloxane as an alternative in the endoscopic treatment of vesicoureteral reflux. J Urol, 1994. 152(4): 1221-4.

274. Dewan, P., Re: Outcome of endoscopic treatment for vesicoureteral reflux in children using polydimethylsiloxane. J Urol, 2003. 169(6): 2303; author reply 2303.

275. Henly, D.R., D. Barret, and T.L. Weiland, Particulate silicone for use in periurethral injections:Local tissue effects and search for migration. J Urol, 1995. 153:. 2039.

276. Atala, A., C.A. Peters, A.B. Retik, and J. Mandell, Endoscopic treatment of vesicoureteral reflux with a selfdetachable balloon system. J Urol, 1992. 148(2 Pt 2): 724-7.

277. Merguerian, P., G. McLorie, and A. Khoury, Submucosal injection of polyvinyl alcohol foam in rabbit bladder. $J$ Urol, 1990. 144:. 531.

278. Wheeler DM, Vimalachandra D, Hodson EM, Roy LP, Smith GH, Graig JC. Intervenciones para el reflujo vesicoureteral primario. En: La Cochrane Library en español. Oxford: Update Software.

Dr. E. de la Peña Zarzuelo

Servicio de Urología. Fundación Hospital Alcorcón

Budapest, 1 - 28922 Alcorcón (Madrid)

E-mail: epenaz@fhalcorcon.es

(Trabajo recibido el 25 octubre de 2004) 